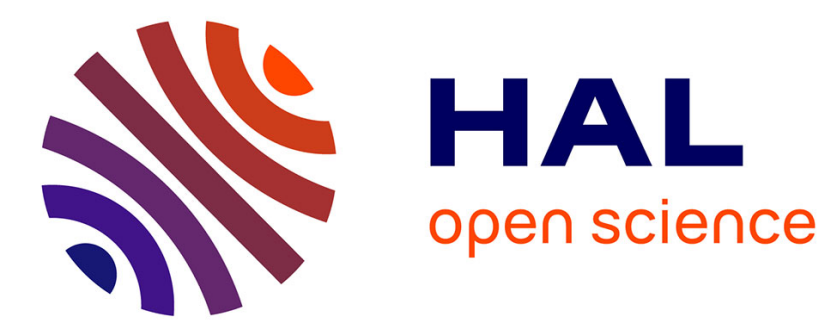

\title{
Efficiency of bio- and socio-inspired optimization algorithms for axial turbomachinery design
}

Mohamed Abdessamed Ait Chikh, Idir Belaidi, Sofiane Khelladi, José Paris, Michael Deligant, Farid Bakir

\section{- To cite this version:}

Mohamed Abdessamed Ait Chikh, Idir Belaidi, Sofiane Khelladi, José Paris, Michael Deligant, et al.. Efficiency of bio- and socio-inspired optimization algorithms for axial turbomachinery design. Applied Soft Computing, 2018, 64, pp.282-306. 10.1016/j.asoc.2017.11.048 . hal-02445287

\section{HAL Id: hal-02445287 \\ https://hal.science/hal-02445287}

Submitted on 20 Jan 2020

HAL is a multi-disciplinary open access archive for the deposit and dissemination of scientific research documents, whether they are published or not. The documents may come from teaching and research institutions in France or abroad, or from public or private research centers.
L'archive ouverte pluridisciplinaire HAL, est destinée au dépôt et à la diffusion de documents scientifiques de niveau recherche, publiés ou non, émanant des établissements d'enseignement et de recherche français ou étrangers, des laboratoires publics ou privés. 


\title{
Efficiency of bio- and socio-inspired optimization algorithms for axial turbomachinery design
}

\author{
Mohamed Abdessamed Ait Chikh ${ }^{\mathrm{a}, *}$, Idir Belaidi ${ }^{\mathrm{a}}$, Sofiane Khelladi ${ }^{\mathrm{b}}$, José Paris ${ }^{\mathrm{c}}$, \\ Michael Deligant ${ }^{\mathrm{b}}$, Farid Bakir ${ }^{\mathrm{b}}$ \\ a Laboratoire d'Energétique, Mécanique et Ingénierie, Université de Boumerdes, Algeria \\ b Laboratoire de Dynamique des Fluides, Arts et Métiers ParisTech, France \\ c GMNI, Universidad da Coruña, Spain
}

\begin{abstract}
A B S T R A C T
Turbomachinery design is a complex problem which requires a lot of experience. The procedure may be speed up by the development of new numerical tools and optimization techniques. The latter rely on the parameterization of the geometry, a model to assess the performance of a given geometry and the definition of an objective functions and constraints to compare solutions. In order to improve the reference machine performance, two formulations including the off-design have been developed. The first one is the maximization of the total nominal efficiency. The second one consists to maximize the operation area under the efficiency curve. In this paper five optimization methods have been assessed for axial pump design: Genetic Algorithm (GA), Particle Swarm Optimization (PSO), Cuckoo Search (CS), Teaching Learning Based Optimization (TLBO) and Sequential Linear Programming (SLP). Four non-intrusive methods and the latter intrusive. Given an identical design point and set of constraints, each method proposed an optimized geometry. Their computing time, the optimized geometry and its performances (flow rate, head $(H)$, efficiency $(\eta)$, net pressure suction head (NPSH) and power) are compared. Although all methods would converge to similar results and geometry, it is not the case when increasing the range and number of constraints. The discrepancy in geometries and the variety of results are presented and discussed. The computational fluid dynamics (CFD) is used to validate the reference and optimized machines performances in two main formulations. The most adapted approach is compared with some existing approaches in literature.
\end{abstract}

\section{Introduction}

Turbomachines are omnipresent in everyday domestic environment and in industry. The applications range from air conditioning to water distribution and from automotive to aeronautics and naval engines. In the turbomachines design optimization, because of the large number of variables of design parameters, which is in this case always larger than the number of equations, this can be handled by the classical design methods based on the exploitation of empirical laws like Coridier's diagram [1,2].

Most studies concerning turbomachines optimization problem focus on the maximization of efficiency. Some optimization approaches are based on the resolution of analytical and loss models [3,4], others on empirical approaches [5], but both using

\footnotetext{
* Corresponding author.

E-mail address: m.aitchikh@univ-boumerdes.dz (M.A. Ait Chikh).
}

local optimization methods. On such methods, the performances of turbomachines were able to be improved by making geometry modifications. However, it is established that the fluid flow in turbomachines is viscous, three-dimensional (3D) and turbulent. Sorensen et al. [6,7] coupled the resolution of the momentum and the energy differential equations (CFD) with a nonlinear optimization method to optimize the performance of a given design. Moreover, the use of CFD tools within 3D RANS solvers are widely employed with evolutionary optimization algorithms, such as genetic algorithms [8,9], with gradient methods [10] such as Simplex, with simulated annealing (SA) [11] and with differential evolution algorithm [12]. As drawback, the use of CFD for the optimization of turbomachines remains very expensive which directed the researches to the exploitation of alternative methods that establish a relation between the design parameters and the targeted performances. One can make reference to design of experiment methods, response surface, kriging, neuron networks and so on. In using local method optimization with a complex 
problem. The missing of the global solution often happens. May this type of method gives a good precision result when it's coupled with a global method as Genetic Algorithm [13]. Although the latter is considered as global method but the optimal solution is not granted mainly with large dimension of the problem. May be using the other method in parallel, tell us more about optimal solution [14]. This work maybe considered among one of the rare study that compared several optimization methods for turbomachinery design namely GA,SA and Sequential Quadratic Programming. However, the comparison study was not presented as statistical significance. Recently, the PSO algorithm became included in the turbomachinery design specifically in aerodynamics type $[15,16]$. For more information about design optimization methods in turbomachinery aerodynamics, see Ref. [17]. Concerning the optimization strategy and objective parameters, Li [18] proposed a two stages approach to optimize the required Net Positive Section Head (NPSHr) of an axial pump. The optimization is carried out by applying the response surface method with the radial equilibrium equation and the theory of actuator disks. Although this study have allowed to reduce the NPSHr by $37 \%$ under specific required point, the operation pump under other conditions (volumetric flow rate range) was not predicted. Lin et al. [19] proposed a complete approach to minimize the losses of an axial fan and to reduce the overall computational time. This approach has four stages: (1) construction of 3D blade using the "Generated Machining Method", (2) analysis of the performances of the obtained geometry by CFD, (3) establishment of the relation between the geometrical parameters and flow properties by neural networks method, and finally (4) search for the optimal geometry by complex optimization method. These four steps seem long and no reduction of process's time was mentioned. In most cases, the objective of the design of an axial fan/pump aims at the optimization of the efficiency. Huang and Gau [20] opted for a different approach, such as the chosen cost function leads to obtaining the desired of volumetric flow rate of an axial fan, since the optimization of efficiency does not guarantee the increase of the volumetric flow rate. They used CFD to estimate the volumetric flow rate according to optimization variables. Starting from an initial geometry, the updates of the design variables were made by Levenberg-Marquardt method to minimize the objective function.

Always concerning axial fans, Lee et al. [21] proposed a strategy for the optimization of an axial fan consisting in coupling experimental analysis with inverse design approach (using TURBOdesign code) for the choice of the decision variables. The response surface method was then used for the optimization of the efficiency and the pressure rise. In another article, Lee et al. [22] presented a numerical procedure of a low rotational speed axial fan optimization. The objective function was established from a polynomial interpolation based on the response surface method and CFD. The method of gradient was then used to find out the optimal shape of the machine. The CPU time required was approximately $12 \mathrm{~h}$ with a Pentium-IV, 3.0 GHz processor. Song and Sun [23] developed an integral design approach of a transonic fan. In order to define an objective function and then optimize the efficiency and the pressure, they combined global optimization method, experience design method, CFD and finally kriging reconstruction. The total running time is about $71 \mathrm{~h}$ with using Dell 7500 (with 2 Xeon $2.93 \mathrm{GHz}$ processors and $24 \mathrm{~GB}$ RAM).

Most works that use modeling of the relationship between design variable and machine's performance are unconstrained or with a low number of constraints, may be because the expensive computational time needed, also, the off-design still using only in the verification of the performance and does not exploit in the optimization process.

Differently to the optimization methods described above, in which the objective functions are conventional "without inte- grating the off-design", the number of constraint is low and the computational time does not represent a priority criterion, we propose in this paper an approach for the design optimization of an axial pump based on inverse method with some meta-heuristics algorithms based on Bio-inspired approaches namely Genetic Algorithm (GA), Particle Swarm Optimization (PSO) and Cuckoo Search (CS), in one hand, and socio-inspired approaches namely Teaching Learning Based Optimization (TLBO). These methods present the advantage to be non-intrusive to the design codes. For each method, many runs are repeated for choosing the best solution.

In this paper, starting from a reference axial pump, we analyze the performance of several natural inspired optimization algorithms in two main formulations of the design problem by considering a very large number of geometrical and mechanical constraints handled by modified constant penalty technique for the reducing CPU time. The first formulation consists of maximizing the total nominal efficiency. Five scenarios of different complexity have been considered using free vortex (FV), constant vortex (CV) and forced vortex (FCV).

In the second formulation, we propose a new objective function which consist in maximizing the surface constructed by the total efficiency and the $[-25 \%,+25 \%]$ range of nominal volume flow rate, with the constraint that the variation of the pump head within this range does not exceed $15 \%$ of that of the specifications. In this formulation, three scenarios are considered using constant vortex strategy. A SLP, which is considered as an intrusive, is also used for first scenario in case one in order to compare the efficiency and the performance of the proposed algorithms to intrusive approaches family algorithms. The computational fluid dynamics (CFD) is employed to predict the performance of the reference and optimized machines. Finally, after the behavior of each algorithm was analyzed and the approaches were classified, the most adapted approach is selected to compare its optimized machines with some existing machines in literature.

\section{Design of axial pumps and fans}

\subsection{Inverse and direct approaches}

The inverse design is applied to define the geometry of the rotor from a priori known specifications (volume flow rate, head and rotational velocity), and geometrical parameters (inlet/outlet hub/tip radius and rotor blades number). The direct approach consists on analyzing the performances of a given geometries, as for instance those given by the inverse method. It permits to define the overall characteristics (performances) of the machine, in other terms, the evolution of head, efficiency, power, NPSH, etc. with flow rate.

The inverse approach uses laws based on a simplified radial equilibrium (1D) model to define some coherent geometries, whereas direct method uses loss laws (incidence, friction, flow leakage, etc.) to estimate the performances of the defined geometry, see Refs. [24,25] for more details concerning the chosen approach. Below some parameters which used in the present inverse design approach:

- The type of vortex is chosen arbitrarily with a hydraulic efficiency $\left(\eta_{H}\right)$ of $70 \%$.

- The rotor outlet tangential velocity $C_{u 2}(r)$ is variable and depends on the radius (between hub and tip) according to Eqs. (1)-(3) for free vortex, constant vortex and forced vortex respectively.

$$
\begin{aligned}
& C_{u 2}(r)=\frac{K}{r} \\
& C_{u 2}(r)=K
\end{aligned}
$$




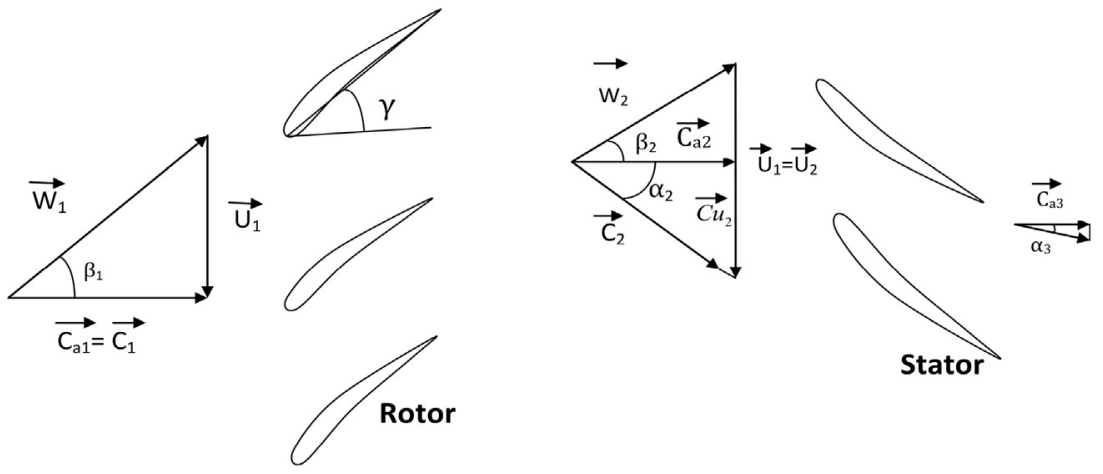

Fig. 1. Velocity triangles of the axial compressible rotor.

$C_{u 2}(r)=K r$

where $K=g H /\left(\omega \eta_{H}\right)$ for free vortex, $K=g H /\left(\omega \eta_{H}\left(R_{i}^{2}+R_{e}^{2}\right) / 2\right)$ for constant vortex and $K=g H /\left(\omega \eta_{H}\left(\left(R_{i}^{2}+R_{e}^{2}\right) / 2\right)^{2}\right)$ for forced vortex, $\mathrm{g}$ is the gravity, $H$ is the head, $\omega$ is the angular velocity and $\left(R_{i}^{2}, R_{e}^{2}\right)$ are the hub and tip outlet radius respectively.

- The velocity triangles are calculated by applying Eulers' equation for each section along the rotor hub and tip radius (radial discretization) (Fig. 1).

- The stagger angle $\gamma$ is determined from the angle of incidence $i$ defined experimentally by Eqs. (4)-(6) $\left(\Delta \beta=\beta_{1}-\beta_{2}\right)$ represent the angular deviation. It is, in fact, given by minimizing losses (continuity of pressure distribution on the blade pressure side) for the case of a blade cascade of NACA65 airfoil (10\% thickness).

$\gamma=\beta_{1}-i$

$i=\frac{\Delta \beta+0.94}{q\left(\beta_{1}\right)}+2.07$

$q\left(\beta_{1}\right)=2.103-4.01910^{-7} \times \beta_{1}^{3.382}$

- The solidity $\left(\sigma^{2}\right)$ at the outlet of the rotor in the hub and tip is expressed according to the diffusion factor of Lieblein $D$, according to Eq. (7) and at the intermediate radius according to Eq. (8) respectively.

$\sigma^{2}=\frac{\cos \left(\beta_{1}\right) \times\left|\mathrm{Ca}_{1} \times \tan \left(\beta_{1}\right)-\mathrm{Ca}_{2} \times \tan \left(\beta_{2}\right)\right|}{2 \times \mathrm{Ca}_{1} \times\left(D-1+\mathrm{Ca}_{2} \times \cos \left(\beta_{1}\right) /\left(\mathrm{Ca}_{1} \times \cos \left(\beta_{2}\right)\right)\right)}$

- The chord at the rotor hub and tip are calculated using Eq. (8) and by linearization at the intermediate radius.

$C=\sigma^{2} \frac{2 \pi r}{Z}$

- The solidity $\left(\sigma^{1}\right)$ at the inlet of the rotor is expressed according to the chord, blades number and the radius, according to Eq. (9).

$\sigma^{1}=\frac{Z C}{2 \pi r}$

- The camber coefficients are expressed according to incidence angle and the inlet solidity, and they are given by Eqs. (10) and (11).

$C_{z \infty 0}=\frac{i+2.525}{p\left(\sigma^{1}\right)}-0.823$

$p\left(\sigma^{1}\right)=15.535-12.467 e^{-0.4242 \sigma^{1}}$

- Finally, to complete the empirical equations relative to the NACA65 airfoil, the solidity and the camber are bounded due to geometrical and technological integrity limitation, and will be used as constraints in the formulation of the optimization problems.

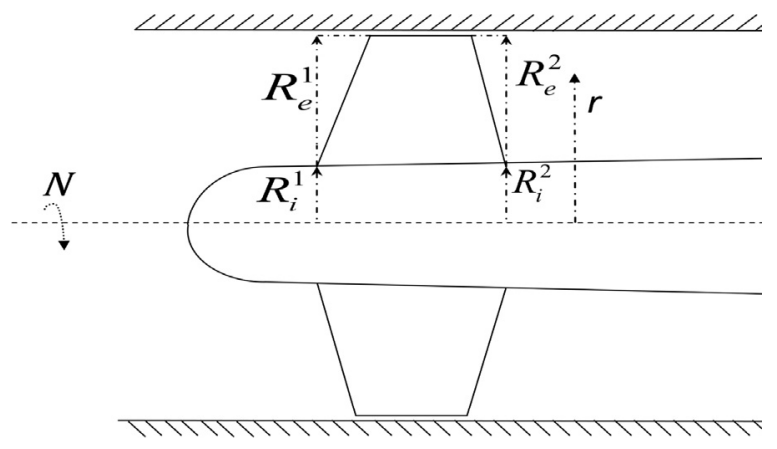

Fig. 2. Scheme of an axial rotor.

\subsection{Design descriptive parameters}

The definition of the geometry of turbomachines requires the knowledge of a set of some geometrical parameters, every valuable variation of parameters describe a different geometrical shape, i.e., a different machine with its own performances. These geometrical parameters will be associated with some other relevant mechanical and fluid parameters.

\subsubsection{Geometrical parameters}

The geometrical parameters considered in the context of this study are: the slack distance between the tip of the rotor blades and the hull, the mean roughness of the rotor blades, the number of the rotor blades $(Z)$, the hub and tip inlet radius $\left(R_{i}^{1}, R_{e}^{1}\right)$, the hub and tip outlet radius $\left(R_{i}^{2}, R_{e}^{2}\right)$, the diffusion factors at the hub $\left(D_{i}\right)$ and the tip $\left(D_{e}\right)$ of the rotor blades, and the minimum value of the maximum thickness of an airfoil section, see Fig. 2.

\subsubsection{Mechanical parameters}

The token fluid properties, are the fluid density $(\rho)$, and the kinematic viscosity of the fluid $(v)$. The mechanical parameters are: the (initial) theoretical volume flow rate $\left(Q_{v}\right)$, rotational velocity $(N)$, head $(H)$.

\section{Formulation of the design optimization problem}

In this section, a model adapted to optimization methods is proposed, it allows to obtain an optimal geometry according to a given objective and needs respecting some imposed constraints.

\subsection{Parameters held constant during the process of optimization}

During all the process of optimization some parameters will be considered constant, for instance: 


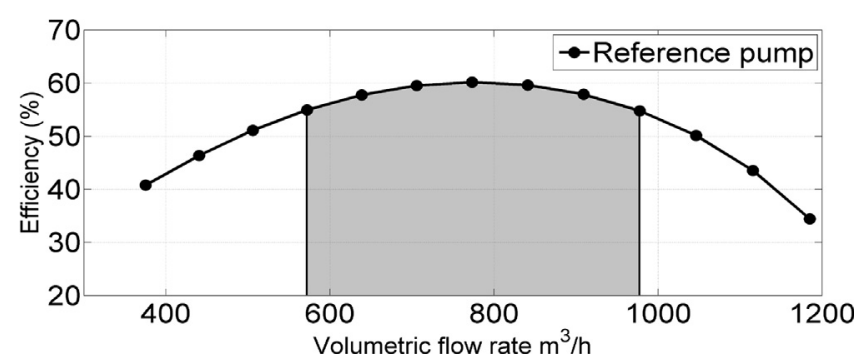

Fig. 3. Objective function of the two cases.

- the slack distance between the rotor blades tip and the hull, the roughness of the rotor blades, the maximal thickness of the rotor blades;

- the fluid properties (density and viscosity),

- parameters connected to the mechanical and fluid properties, being a part of a tender specifications (operating condition needs) such as: the rotational speed, the head, etc. are fixed a priori;

- computational parameters such as the number of volume flow rate point and radial sections discretization, etc. are also considered constant during all the process of optimization. Their value has influence only on the accuracy of the results of the analysis model. These two parameters can be however, modified for more precision.

As already mentioned previously, the modification of this set of parameter allows to obtain several geometrical configurations (several machines with their own corresponding performances).

\subsection{Considered decision variables}

The design variables relative to the proposed model of optimization are considered as real numbers except the number of blades which is taken as integer. Decision variables are: the theoretical volume flow rate $\left(Q_{v}\right)$, inlet hub and tip radius $\left(R_{i}^{1}, R_{e}^{1}\right)$, outlet hub and tip radius $\left(R_{i}^{2}, R_{e}^{2}\right)$, hub and tip diffusion factors $\left(D_{i}, D_{e}\right)$, the number of blades $(Z)$; however, for the local optimization method, the optimal number of rotor blades is determined after analyzing the optimum design of turbomachine with various rotor blade number.

Practically, each design variable has a variation interval (max and min), usually named "side constraints". This interval is defined according to the designer's needs and for other theoretical or environmental constraints. Contrary to classical optimization methods, the meta-heuristics methods using in this paper are based on population constituted of individual solutions, each individual is defined by a set of decision variables.

\subsection{Objective function}

\subsubsection{Non-intrusive methods}

First case. In this first case, the optimization process consists on maximizing the total efficiency on the axial turbomachine (pump/fan) by varying a set of decision variables. In fact, the efficiency depends on many parameters, but generally, the overall performance of the machine is described according to the volume flow rate. A simple design on a range of $\left[Q v_{\min }, Q v_{\max }\right]$ discretized uniformly (Fig. 3) shows that the efficiency is a continuous function having a maximal point corresponding to a value of the specific volume flow rate called: "nominal" volume flow rate.

$\max F=\left(\eta_{\text {nom }}\right)$

Second case. In the second case we propose a new objective function in the field of turbomachine optimization. The purpose of this function is to maximize the area formed by the curve of effi-

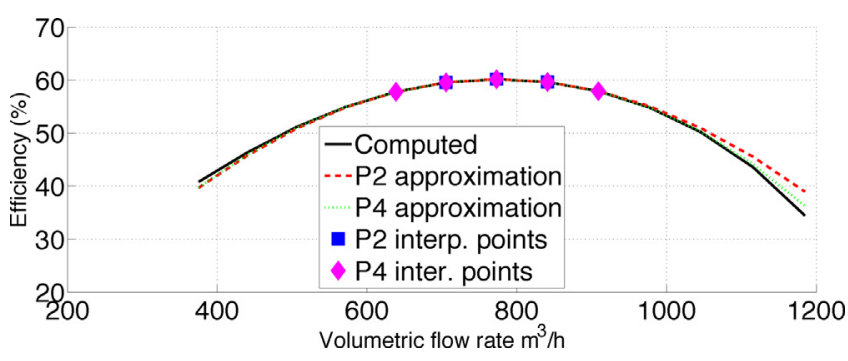

Fig. 4. Comparison of the computed efficiency with a second order interpolating polynomial and a fourth order interpolating polynomial for different values of the volume flow rate.

ciency and the axis of volume flow rate as shown in Fig. 3. The objective of this optimization approach is to widen the range of variation of the efficiency in the neighborhood of the design point (nominal flow condition), a numerical integration (based on trapezoidal rule) is used. The flow rate integration range varies between $-25 \%$ and $+25 \%$ of the nominal volume flows rate (Qvnom), the objective function is then given by:

$\max , F=\int_{0.75 Q_{v n o m}}^{1.25 Q_{\text {vnom }}} \eta\left(Q_{V}\right), d Q_{v}$

\subsubsection{Intrusive method}

The analysis formulation developed by the authors computes the nominal flow rate by studying the response of the proposed design for a set of initial theoretical volume flow rates homogeneously distributed in the interval [Qvmin, Qvmax] previously defined. Consequently, a predefined number of database points of the efficiency-volume flow rate function can be obtained and an approximation to the nominal flow rate can be estimated.

In this paper the authors propose the use of a second order interpolating polynomial to obtain the nominal volume flow rate and the related nominal efficiency. This approximation can be obviously improved by increasing the order of the polynomial but, due to practical considerations, second order has been considered by the authors as adequate for this problem. Some numerical tests have been developed in order to verify this assumption.

Fig. 4 shows the computed values of the efficiency of an initial design of a turbomachine and two interpolating polynomials: a second order interpolating polynomial and a fourth order interpolating polynomial. The complete database is defined by 25 points that corresponds to 25 values of the flow rate homogeneously distributed in the interval [Qvmin, Qvmax]. The second order interpolating polynomial is defined by three database points: the volume flow rate with maximum efficiency and the two neighbor volume flow rates studied. The fourth order interpolating polynomial is defined by five database points: the volume flow rate of the database with maximum efficiency and the first and second neighbors. According to this figure, both approximations are adequate in an interval centered at the volume flow rate that produces maximum efficiency, where the nominal volume flow rate and nominal efficiency are contained. The computed values, assumed as exact in this analysis, are obtained by using a larger database with 100 discrete values of the volume flow rate homogeneously distributed in the interval [Qvmin, Qvmax]. In practice, the relative error of the approximation around the nominal volume flow rate is adequate for this problem.

Fig. 5 shows the computed values of the efficiency and two interpolating functions: a second order interpolating polynomial and a fourth order interpolating polynomial. Both of them are obtained with the same algorithms used in the first study example. According to this figure, both approximations are also adequate in an interval centered at the maximum value of the efficiency that contains the nominal volume flow rate and the related nominal efficiency. 


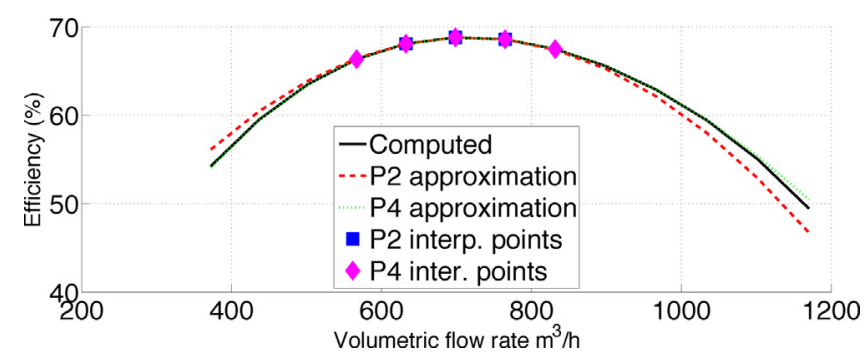

Fig. 5. Comparison of the computed efficiency with a second order interpolating polynomial and a fourth order interpolating polynomial for different values of the volume flow rate.

Table 1

Nominal volume flow rate and nominal efficiency for a initial design of a turbomachine and comparison with the second order polynomial interpolation and the fourth order polynomial interpolation.

\begin{tabular}{lll}
\hline Initial design & $Q v_{\text {nom }}\left(\mathrm{m}^{3} / \mathrm{h}\right)$ & $\eta_{\text {nom }}(\%)$ \\
\hline Computed values & 776.9440 & 60.15808 \\
Second order polynomial approx & 776.8716 & 60.15801 \\
Relative error & $9.31 \times 10^{-4}$ & $1.20 \times 10^{-6}$ \\
Fourth order polynomial approx & 776.9515 & 60.15809 \\
Relative error & $9.65 \times 10^{-5}$ & $3.86 \times 10^{-8}$ \\
\hline
\end{tabular}

Table 2

Nominal volume flow rate and nominal efficiency for an improved design of a turbomachine and comparison with the second order polynomial interpolation and the fourth order polynomial interpolation.

\begin{tabular}{lll}
\hline Improved design & $Q v_{\text {nom }}\left(\mathrm{m}^{3} / \mathrm{h}\right)$ & $\eta_{\text {nom }}(\%)$ \\
\hline Computed values & 719.9224 & 68.68955 \\
Second order polynomial approx & 720.0684 & 68.68900 \\
Relative error & $2.03 \times 10^{-4}$ & $8.00 \times 10^{-6}$ \\
Fourth order polynomial approx & 719.90792 & 68.68955 \\
Relative error & $2.01 \times 10^{-5}$ & $1.39 \times 10^{-8}$ \\
\hline
\end{tabular}

The computed values, assumed as exact in this analysis, are also obtained by using a larger database with 100 discrete values of the initial volume flow rate homogeneously distributed in the interval [Qvmin, Qvmax].

Figs. 4 and 5 and Tables 1 and 2 show that the proposed second order interpolating polynomial gives accurate approximations of the nominal volume flow rate and nominal efficiency.

The objective function of this problem is the maximization of the nominal efficiency. Thus, we propose to use the second order interpolating polynomial as the mathematical expression that allows to compute the objective function. In practice, the nominal volume flow rate can be obtained as:

$Q v_{\text {nom }}=\frac{\frac{S_{i-1}}{P_{i-1}} \eta_{i-1}+\frac{S_{i}}{P_{i}} \eta_{i}+\frac{S_{i+1}}{P_{i+1}} \eta_{i+1}}{2\left(\frac{\eta_{i-1}}{P_{i-1}}+\frac{\eta_{i}}{P_{i}}+\frac{\eta_{i+1}}{P_{i+1}}\right)}$

being:

$P_{i}=\prod\left(Q v_{i}-Q v_{j}\right), \quad j \in\{i-1, i, i+1\}$

$S_{i}=\sum_{j \neq i}^{j \neq i} Q v_{j}, \quad j \in\{i-1, i, i+1\}$

and $\eta_{j}$ the efficiency of the axial turbomachine related to volume flow rate $Q v_{i}$ being. The volume flow rate $Q v_{i}$ corresponds to the value of the database with the largest value of efficiency. The lower and upper neighbor points of $Q v_{i}$ that defines the second order interpolating polynomial are identified as $Q v_{i-1}$ and $Q v_{i+1}$, respectively. The nominal efficiency related to the nominal volume flow rate can be easily obtained by computing the second order Lagrange interpolating polynomial as:

$$
\begin{aligned}
\eta_{\text {nom }}= & \frac{\left(Q v_{\text {nom }}-Q v_{i}\right)\left(Q v_{\text {nom }}-Q v_{i+1}\right)}{P_{i-1}} \eta_{i-1} \\
& +\frac{\left(Q v_{\text {nom }}-Q v_{i-1}\right)\left(Q v_{\text {nom }}-Q v_{i+1}\right)}{P_{i}} \eta_{i} \\
& +\frac{\left(Q v_{\text {nom }}-Q v_{i-1}\right)\left(Q v_{\text {nom }}-Q v_{i}\right)}{P_{i+1}} \eta_{i+1}
\end{aligned}
$$

Thus, the objective function of the optimization problem consists of maximizing $\eta_{\text {nom }}$ defined according to Eq. (16). In practice, optimization problems are usually stated as minimization problems. Thus, the objective function consists of minimizing:

$\max , F=-\eta_{\text {nom }}$

On the other hand, the optimization of turbomachines also requires some specifications to be satisfied. In practice, these specifications are treated in an optimization problem as design constraints.

\subsection{The retained constraints}

\subsubsection{Side constraints}

In the search for the optimal geometrical shape certain violations of the constraints must be avoided. The constraints of the design variable or the side constraints allow to establish inequalities of the search spaces described below. These spaces represent the first constraint that must be treated, since in the practice, all the design variables must be limited by two values ( $\min$ and max). In the model of optimization all the design variables vary between two predefined positive extremes. We note:

$x_{j, \text { Min }} \leq x_{j} \leq x_{j, \text { Max }} \quad 1 \leq j \leq D$

Being that $D$ is the number of variables or the dimensions of design optimization problems. This type of constraint is handled differently than the other types i.e. without the integration in the fitness of the individual.

\subsubsection{Geometric constraints}

The first inclusive geometrical constraint is the hub radius constraints. Because of the random nature of the meta-heuristics algorithms, it can fall in cases where the hub radius at the entrance of the rotor is larger than that at the exit, which could cause a perturbed flow. A constraint of the entrance and exit hub radius must be respected as follows:

$R_{i}^{1}-R_{i}^{2} \leq 0$

On the other hand, the constraint of the rotor thickness has been added, which represents the distance between the entrance and the exit of the turbomachine. This parameter is calculated in every radius situated between hub and tip of the rotor. Every section of the discretization has a different airfoil geometry (the stagger angle, the chord length, the camber, the thickness of airfoil. etc.), all these geometries of airfoil along the radial direction define the blade of the turbomachine. In each section, the thickness of the rotor and the chord form a stagger angle. The thickness is the projection of the chord on the longitudinal axis. The design of the turbomachine must satisfy that a predefined maximal thickness should not be exceeded. This constraint is represented mathematically as follows:

$l_{r} \cos \left(\gamma_{r}\right)-t h_{\max } \leq 0, \quad r=1, \ldots, N_{r d}$

where that $l_{r}$ and $\gamma_{r}$ represent respectively the chord and the stagger angle to each section $r$; $t h_{\max }$ the maximum rotor thickness allowed, $N_{r d}$ is the number of discretization sections.

This model would need local constraints due to empirical reasons linked directly with the airfoil shape of the chosen blade. 
Consequently, a maximum camber value was imposed for each section of the radial discretization.

$C_{z \infty o}^{r}-2.7 \leq 0$

The same reasons for the solidity in the entrance $\sigma_{r}^{1}$ And at the exit $\sigma_{r}^{2}$ of the rotor.

$\sigma_{r}^{1}-1.5 \leq 0$ and $\sigma_{r}^{2}-1.5 \leq 0$

To ensure geometric relevance and to avoid any kind of inadequate shapes of the optimized rotor blade. The inlet flow angle must be greater than the exit flow angle on all radially discretized sections.

$\beta_{1}^{r}-\beta_{2}^{r}>0$

Finally a constraint of minimum nominal value of volume flow rate was considered to guarantee the specifications of the turbomachine.

\subsubsection{Mechanical constraint}

To complete the constraints, the nominal volume flow rate must be limited by a lower value. By the fact that the problem of optimization consists of reducing the nominal volume flow rate in order to increase the total efficiency.

$Q v_{n o m}-\hat{Q} v \geq 0$

Knowing that $\hat{Q} v$ is the allowed minimum nominal volume flow rate.

The head constraint is added specially for second formulation problem:

$|H(0.75 Q v)-H(1.25 Q v)| \leq 0.15 H\left(Q v_{\text {nom }}\right)$

\section{Constraints handling}

\subsection{Non-intrusive methods}

A review of the literature [26-28] shows that the constraint handling methods can be classified as:

- Methods based on preserving feasibility of solutions;

- Methods based on penalty functions;

- Methods making distinction between feasible and unfeasible solutions;

- Methods based on decoders;

- Hybrid methods.

The method of penalty is the oldest one, its difficulty is in choosing the most effective penalty coefficient according to the problem setup. Deb [28] proposed a technique without using the coefficient of penalty, which is based on the principle of feasible and unfeasible solutions.

In our case (single-objective optimization), as mentioned above, the total efficiency needs to be improved in the interval [0,1]. Like the other performances parameters, where their maximum and minimum values can be estimated, the coefficient of penalty must be satisfied in order to penalize the objective function (the fitness moves outside its theoretical interval).

The variable constraints (side constraints) are treated differently. The constraints do not influence on the fitness. That means that there is no penalization of the objective function. Nevertheless, the constraints must be respected during the evolution of the population.

Other types of constraints may be handled by using the technique of static penalty function [29]. This technique consists of penalizing the solutions located in the unfeasible region by using a penalty constant.

fitness $=F+\sum_{i=1}^{m} C_{i} \delta_{i}$

where $\left\{\begin{array}{l}\delta_{i}=1 \text { if constraint is not respected } \\ \delta_{i}=0 \text { else }\end{array}\right.$

where $m$ is the number of constraints, $C$ the constant of penalty depending on the objective function $F$.

In this paper, we propose a strategy that handles the constraints in a way that it decreases the computational time as much as possible. The evaluation of the individual begins with the inverse design to determine the geometrical parameters of the machine, just before passing to the direct method for analyzing the performances, one verifies the geometrical constraints (camber, solidity, blade thickness, etc.). The fitness of the individual is initialized by null value, if there is at least a single violation of the constraints, the individual will be penalized by a constant of penalty equal to 1. In this case, it is not necessary to analyze the machine by the direct method. If the opposite occurs, i.e. no violation of the constraints is found, one passes to the direct method to determine the main fitness which is the nominal total efficiency. The nominal constraint of minimum volume flow rate is then handled with the same method of penalty. The elimination of the direct method for individuals who do not verify the geometrical constraints allows to decrease dramatically the computational time.

\subsection{Intrusive methods}

The handling of the constraints in the intrusive gradient-based methods is strongly linked to the design method itself, this phase will be detailed in the algorithmic development section.

\section{Optimization algorithms}

Four bio- and socio-inspired meta-heuristics are used in this work:

- bio-inspired: Genetic Algorithm (GA), Particle Swarm Optimization (PSO), Cuckoo Search (CS);

- socio-inspired: Teaching Learning Based Optimization (TLBO).

The proposed set of methods are iterative and non-intrusive, the variables are modified in each iteration.

\subsection{Genetic Algorithm}

\subsubsection{General principle}

The well known Genetic Algorithms are a part of the evolutionary family based on the theory of evolution and natural selection proposed by Darwin. The method, developed by Holland (since 1960), simulates mathematically the operators of crossover, mutation and selection [30]. GA was made popular by Goldberg [31]. An individual (set of variables) is represented by a chromosome and a gene represents a variable formed by a string of 0 and 1. Binary form of representing variable is the most used.

In this work, real form of variables is used, the flexibility and the performances of the algorithm in terms of speed of execution are better [32].

\subsubsection{GA operators}

5.1.2.1. Selection. We chose the selection by binary tournament. Two individuals are randomly pulled $x_{1}$ and $x_{2}$, and the one who 
has the best fitness will be in the next generation $(t+1)$, the other one will be rejected.

if $\left(\right.$ fitness $\left.\left(x_{1}^{t}\right)>\operatorname{fitness}\left(x_{2}^{t}\right)\right) \quad x^{t+1}=x_{1}^{t}$;

else $x^{t+1}=x_{2}^{t}$;

where,

$1 \leq t \leq$ iteration, $\max$

5.1.2.2. Crossover. The intermediate crossover, proposed by Kaya et al. [33], which allows to create two "children" from two randomly pulled "parents" is used, this crossover is controlled by a ratio.

$x_{1}^{t+1}=x_{1}^{t}+$ rand $\times$ ratio $\times\left(x_{2}^{t}-x_{1}^{t}\right)$

$x_{2}^{t+1}=x_{2}^{t}+$ rand $\times$ ratio $\times\left(x_{2}^{t}-x_{1}^{t}\right)$

where rand is a random number between $[0,1]$ and the ratio is a constant between $[0,1]$, it can be larger than 1 if there is a problem of premature convergence; ratio $=1.2$.

5.1.2.3. Mutation. A Gaussian mutation [34] is preferred with a probability of $P m=0.01$. This method adds a random normal distribution for each variable.

$x_{i d}^{t+1}=x_{i d}^{t}+S \times \operatorname{rand}_{n} \times\left(x_{d, \min }-x_{d, \max }\right)$

$S=$ scale $\times\left(\frac{1-\text { shrink } \times(t+1)}{t_{\max }}\right)$

with,

$1 \leq i \leq n,($ Population, size)

$1 \leq d \leq D$, (number, of , design, variables)

$x_{d \min }, x_{d \max }$ are respectively the minimum and the maximum values of variable $x_{d}$. Knowing that scale is a parameter which determines a standard deviation of the generated random number, its value is between $[0,1]$, shrink is a number between $[0.5,1.0]$; in our case we took scale $=0.1$ and shrink $=0.5$.

\subsubsection{Implementation}

Each decision variable $x_{i d}$ represents a design variable:

$x_{i d} \in\left[Q_{v}, N, R_{i}^{1}, R_{e}^{1}, R_{i}^{2}, R_{e}^{2}, D_{e}, D_{i}, Z\right]$

Algorithm 1 shows GA optimization process of an axial pump.

Algorithm 1. Design approach using Genetic Algorithm

\begin{tabular}{|c|c|}
\hline 1: & Start \\
\hline \multirow[t]{5}{*}{ 2: } & Input parameters: \\
\hline & $\begin{array}{l}\text { - Pa, side constraints, maximum iteration, population size, } \\
\text { number of dimensions (variable) }\end{array}$ \\
\hline & $\begin{array}{l}\text { - Design constant: range of volume flow rate, the head } H \text {, } \\
\text { rotational speed } N \text { the density and the viscosity of fluid the }\end{array}$ \\
\hline & roughness, the slack distance between the tip of the rotor \\
\hline & $\begin{array}{l}\text { blades and the hull, the maximum thickness of rotor, type of } \\
\text { vortex, number of the volume flow rate and the section }\end{array}$ \\
\hline 3: & Initialize the population randomly \\
\hline 4: & Evaluate the population opulation: \\
\hline 5: & - Generate the geometry (inverse design) \\
\hline 6: & - Check the geometrical constraints with the penalty method \\
\hline 7: & if no violation found then \\
\hline 8: & - Analyze the performances (direct design) \\
\hline 9: & $\begin{array}{l}\text { - Check the mechanical constraints (nominal volume flow } \\
\text { rate) with the penalty method (compute the total fitness) }\end{array}$ \\
\hline 10: & end if \\
\hline 11: & for iteration: $=1$ to Max iteration do \\
\hline 12: & Selection Eq. (27) or (28). \\
\hline 13: & Crossover Eqs. (29) and (30) \\
\hline 14: & Mutation Eqs. (31) and (32) \\
\hline 15: & Evaluate the population: \\
\hline
\end{tabular}

\begin{tabular}{ll} 
16: & - Generate the geometry (inverse design) \\
17: & - Check the geometrical constraints with the penalty method \\
18: & if no violation found then \\
19: & Analyze the performances (direct design) \\
$20:$ & Check the mechanical constraints (nominal volume flow \\
& rate) with the penalty method (compute the total fitness) \\
$21:$ & end if \\
$22:$ & end for \\
$23:$ & return The best solution is obtained \\
\hline
\end{tabular}

\subsection{Particle Swarm Optimization}

\subsubsection{General principle}

Particle Swarm Optimization (PSO) method was developed by Kennedy and Eberhart in 1995 [35] it is based on the simulation of the swarm behavior of birds or fishes. A particle or an individual represents a bird/fish who is analogically a set of variable in an optimization problem. When the individual moves from a position to another, it is affected by three main factors:

1. the attraction toward the leader team,

2. the attraction toward its best position so far,

3. staying in the actual position.

$$
\begin{aligned}
& V_{i d}^{(t+1)}=w \times V_{i d}^{(t)}+C_{1} \times \text { rand } \times\left(\text { Pbest }_{i d}^{(t)}-x_{i d}^{(t)}\right)+ \\
& C_{2} \times \text { rand } \times\left(\text { Gbest }_{d}^{(t)}-x_{i d}^{(t)}\right)
\end{aligned}
$$

$x_{i d}^{(t+1)}=x_{i d}^{(t)}+V_{i d}^{(t+1)}$

Knowing that Pbest is the best position passed by the individual, Gbest (the best global individual), is the best position of all best positions of the generation, $V$ is the velocity of the decision variable, $C_{1}=C_{2}=2.0$ are cognitive coefficient and social coefficient respectively, $w$ is the inertia weight, it can be fixed to 0.5 or varied between 0.4 and 0.9 during iterations. The inertia weight can be given by,

$w^{(t)}=w_{\min }+\left(\frac{t_{\max }-t}{t_{\max }}\right) \times\left(w_{\max }-w_{\min }\right)$

where $w_{\min }$ and $w_{\max }$ are the minimums and the maximums values of the inertia weight. This formulation, called Linearly Decreasing Weight Particle Swarm Optimization (LDW-PSO), is proposed by Shi and Eberhart [36]. A literature review shows a lot of approachs proposing improvements like in [37] who developed an automatic restart of the calculation (multi-start PSO) with an initial population created randomly when a stagnation of fitness variation during the iterations occur. Wei et al. [38] add elitism and mutation mechanisms to the PSO. In this paper, we propose to use standard PSO with $w=0.5$ and an unlimited velocity.

\subsubsection{Algorithmic implementation}

For each $x_{i d}$, Pbest $t_{i d}$ and Gbest ${ }_{i d}$ such as:

$\left\{\begin{array}{l}x_{i d} \\ \text { pbest }_{i d} \\ \text { gbest }_{d}\end{array}\right\} \in\left[Q_{v}, N, R_{i}^{1}, R_{e}^{1}, R_{i}^{2}, R_{e}^{2}, D_{e}, D_{i}, Z\right]$ 
Algorithm 2. Design approach using Particle Swarm Optimization algorithm

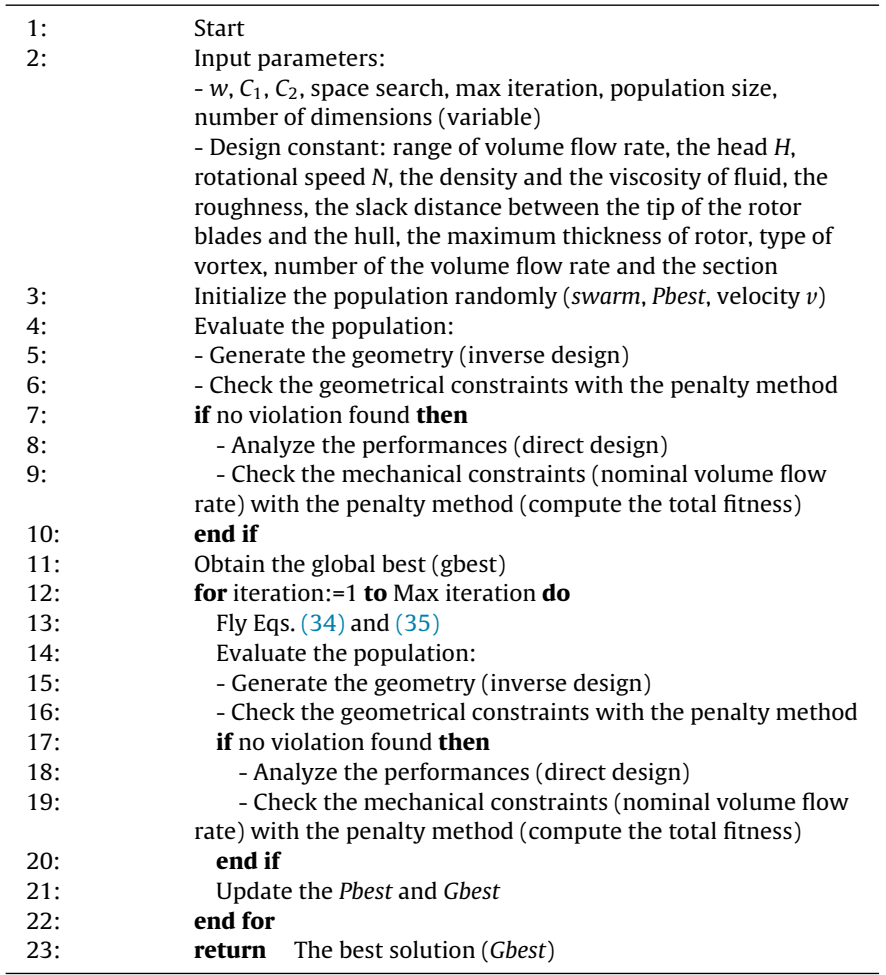

\subsection{Cuckoo Search Algorithm}

\subsubsection{General principle}

Following the example of the previous algorithms, the Cuckoo Search (CS) is a natural-inspired method formulated by Yang and Deb [39]. CS is based on the behavior of cuckoo, which is characterized by the practice of "parasitism of brood", combined to the Levy flight (random walk), which is another behavior of some birds. In this algorithm, an individual (proposed solution or the set of variables) is considered as a "nest". A nest which has a good quality eggs represent an individual of good fitness.

The standard CS algorithm [40] has three main stages:

1. each cuckoo lays an egg at a time and drops it in a randomly chosen nest,

2. the best nest with a high quality of eggs continue existing in the next generation,

3. there is a probability $\mathrm{Pa}$ (between 0 and 1 ), that the host bird discovers the cuckoo eggs, thus he can get rid of it either by abandoning its nest or by reconstructing another.

The phase of choosing a nest is described as follows:

$$
\begin{aligned}
& x_{i}^{(t+1)}=x_{i}^{(t)}+\alpha \oplus \operatorname{Lévy}(\beta) \\
& \alpha=\alpha_{0} \times\left(\text { best }-x_{i}^{(t)}\right) \\
& \alpha_{0} \times\left(\text { best }-x_{i}^{(t)}\right) \oplus \operatorname{Lévy}(\beta) \approx 0.01 \frac{\mu}{|v|^{\frac{1}{\beta}}}\left(\text { best }-x_{i}^{(t)}\right) \\
& \mu \approx N\left(0, \sigma_{\mu}^{2}\right) \\
& v \approx N\left(0, \sigma_{v}^{2}\right)
\end{aligned}
$$$$
\sigma_{\mu}^{2}=\left[\frac{(\Gamma(1+\beta) \sin (\beta \pi / 2))}{\left(\Gamma[(1+\beta) / 2] \times \beta \times 2^{(\beta-1) / 2}\right)}\right]^{\frac{1}{\beta}}
$$

$\sigma_{v}^{2}=1$

where $\mu$ is the normal distribution of an average 0 and a variance $\sigma_{\mu}^{2}, v$ is the normal distribution of an average 0 and a variance 1 .

The phase of changing the nest for the aim of finding a new solution with biased/selective random walks [41] is given as follows:

Two solutions are randomly pulled, $x_{p}$ and $x_{q}$ and a new nest from these two solutions is then looked for, therefore

stepsize $=$ rand $\times\left(x_{p}^{t}-x_{q}^{t}\right)$

new $x_{i}^{t}=x_{i}^{t}+$ stepsize

\subsubsection{Algorithmic implementation}

Every nest represents a set of decision variables $x_{i d}$. The code in Algorithm 3 shows the process of design optimization of an axial pump by CS.

Algorithm 3. Design approach using Cuckoo Search Algorithm

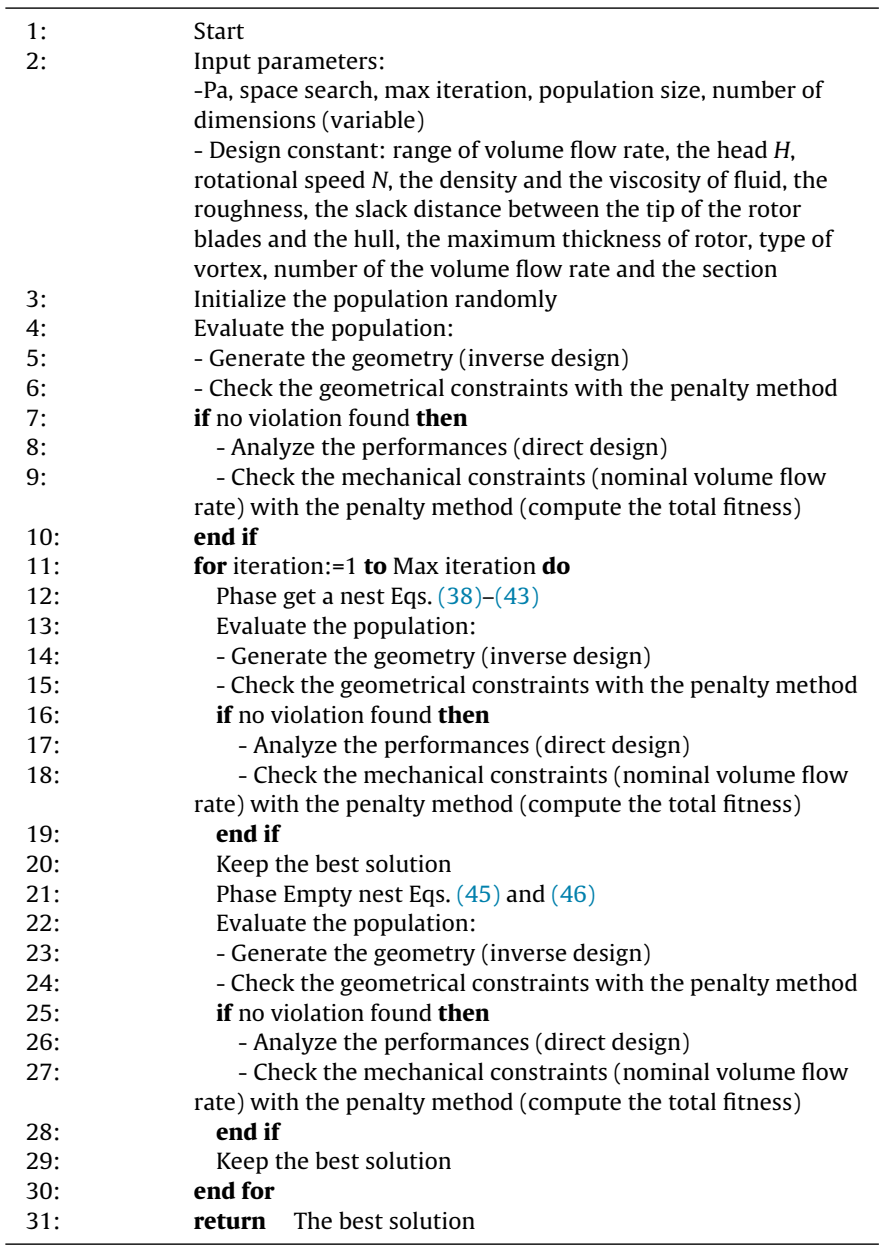

\subsection{Teaching-Learning based Optimization Algorithm}

\subsubsection{General principle}

The previously presented algorithms are natural or bio-inspired. Differently, the Teaching Learning based Optimization algorithm (TLBO) proposed by Rao et al. [42] is based on the influence of a professor on students scholar performances and the influence of students on each other. Therefore, this is why this method is considered as socio-inspired. A population is presented by students, each student represents an individual, that is mean a solution which contains the variables, referred to as subjects. The teacher is the best individual (reference) which can latter be changed during the iterations. Opposed to other algorithms like GA, PSO and CS, where 
the choice of the parameters remains always dependent on several regulation tests, TLBO do not have any regulation parameter. TLBO was tested with several constrained benchmark functions, where it showed good performances [43].

TLBO is characterized by two phases: teaching and learning.

5.4.1.1. Teaching phase. In this phase, the teacher which is the individual who has the best fitness tries to raise or to improve the average of marks of his students (the individuals of the rest of the population) by transmitting its knowledge. A good teacher forms good students. $X_{i}=\left\{x_{i 1}, x_{i 2}, x_{i 3}, \ldots, x_{i D}\right\}$, Xi: individual (student), $x_{i 1}, x_{i 2}, x_{i 3}$ : the decision variables (the subjects) $i=\{1,2,3,4,5, \ldots$, $N\}, N$ is the number of student (population size).

$x_{i}^{(t+1)}=x_{i}^{(t)}+$ rand $\times\left(x_{\text {Teacher }}^{t}-T_{F} M^{t}\right)$

Knowing that $M^{t}$ is a vector mean of each subject.

$M^{t}=\left[m_{1}^{t}, m_{2}^{t}, m_{3}^{t}, m_{4}^{t}, \ldots, m_{D}^{t}\right]$

$T_{F}$ is not a parameter of TLBO algorithm. Several tests with various values of $T_{F}$ showed that the algorithm is more successful when $T_{F}$ take a value between 1 and 2 . The best manner to improve the algorithm is to set $T_{F}$ as a random variable between 1 and 2 during the iterations [44].

5.4.1.2. Learning phase. In this phase, repeatedly two students of the population are pulled randomly, they change knowledge between each other by a mutual interaction. Every student learn new "things" from the other that has more knowledge (having a good fitness). This phenomenon was mathematically described by Rao et al. [42] and expresses as:

$x_{i}^{(t+1)}=\left\{\begin{array}{l}x_{i}^{(t)}+\operatorname{rand} \times\left(x_{i}^{(t)}-x_{j}^{(t)}\right) \text { if } \\ , x_{i}^{(t)} \\ , \text { is } \\ \text { better } \\ \text { than } \\ x_{j}^{(t)} \\ x_{i}^{(t)}+\operatorname{rand} \times\left(x_{j}^{(t)}-x_{i}^{(t)}\right) \text { otherwise }\end{array}\right.$

\subsubsection{Algorithmic implementation}

In this algorithm, each subject $x_{i d}$ represents one design variable, a set of variable gives a student i.e. a solution, the teacher is always the best solution provided by the TLBO. In both phases, during the iterations, it is always necessary to keep the good student and to reject the one who has a low fitness.

The code in Algorithm 4 shows the design optimization process of an axial pump by TLBO.

Algorithm 4. Design approach using Teaching-Learning-Based Optimization Algorithm

\begin{tabular}{ll}
\hline $1:$ & Start \\
$2:$ & Input parameters: \\
& - Space search, max iteration, population size, number of \\
& dimensions(variable) \\
& - Design constant: range of volume flow rate, the head $H$, \\
& rotational speed $N$, the density and the viscosity of fluid, the \\
& roughness, the slack distance between the tip of the rotor \\
& blades and the hull, the maximum thickness of rotor, type of \\
& vortex, number of the volume flow rate and the section \\
& Initialize the population randomly \\
$3:$ & Evaluate the population: \\
$4:$ & - Generate the geometry (inverse design) \\
$5:$ & if no violation found then \\
6: & - Analyze the performances (direct design) \\
8: & - Check the mechanical constraints (nominal volume flow \\
$9:$ & rate) with the penalty method (compute the total fitness)
\end{tabular}

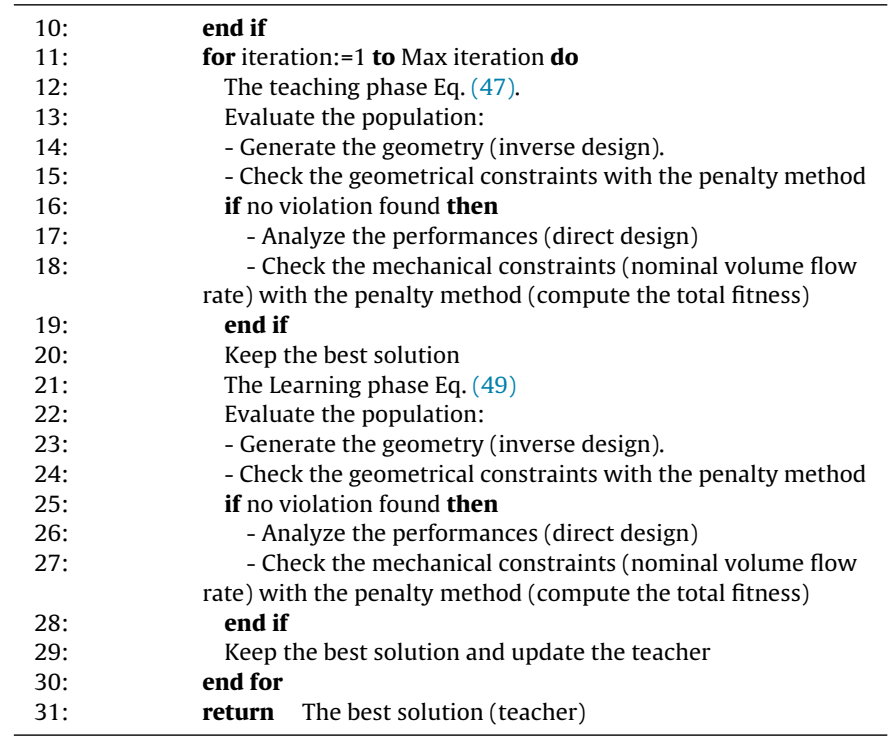

\subsection{Sequential Linear Programming and Simplex}

A mathematical model of the optimization problem has been introduced. According to that, a set of design variables $(x)$ has been defined according to the stated optimization problem. The optimization methodology proposed states an iterative procedure where the design variables are modified at each iteration as:

$x^{k}=x^{k-1}+\Delta x^{k}, \quad k \geq 1 ;$

being $k$ the index of the iteration. Thus, the main goal of the optimization algorithm consists of finding the value of $\Delta x^{k}$ that minimizes the objective function and satisfies the constraints imposed according to the definitions proposed in Sections 3.3 and 3.4. In this paper, the authors propose an optimization algorithm based on the use of linearized approximations. Thus, the objective function can be approximated by using the first order Taylor expansions as:

$F\left(x^{k}\right) \approx F\left(x^{k-1}\right)+\left.\frac{\partial F}{\partial x}\right|_{x^{k-1}} \Delta x^{k}$

Thus, the minimization of the first order approximation can be treated as the minimization of the right hand side term since $F\left(x^{k-1}\right)$ is constant in iteration $k$. On the other hand, the constraints of the optimization model must be also satisfied. Consequently,

$g_{j}\left(x^{k}\right) \leq 0, \quad j=1, \ldots, m$

where $m$ is the total number of constraints. In practice, design constraints are usually nonlinear but, following the same idea used in the approximation of the objective function, first order Taylor expansions are used as:

$g_{j}\left(x^{k}\right) \approx g_{j}\left(x^{k-1}\right)+\left.\frac{\partial g_{j}}{\partial x}\right|_{x^{k-1}} \Delta x^{k}, \quad j=1, \ldots, m$

If second and higher order terms of the Taylor expansions are not considered, the verification of the constraints proposed can be stated as:

$\left.\frac{\partial g_{j}}{\partial x}\right|_{x^{k-1}} \Delta x^{k} \geq-g_{j}\left(x^{k-1}\right)$

The linearized problem can be solved by including slack variables in the design constraints as:

$\left.\frac{\partial g_{j}}{\partial x}\right|_{x^{k-1}} \Delta x^{k}+h_{j}=-g_{j}\left(x^{k-1}\right)$ 
Table 3

Specifications of the pump.

\begin{tabular}{ll}
\hline Characterstic & Pump mono-rotor \\
\hline$H(\mathrm{~m})$ & 6 \\
$Q v_{\min }\left(\mathrm{m}^{3} / \mathrm{h}\right)$ & 360 \\
$Q v_{\max }\left(\mathrm{m}^{3} / \mathrm{h}\right)$ & 1800 \\
$N(\mathrm{rpm})$ & 1500 \\
Density of fluid $\left(\mathrm{kg} / \mathrm{m}^{3}\right)$ & 1000 \\
\hline
\end{tabular}

where $h_{j} \geq 0$ are the slack variables. This algorithm is based on the validity of the first order approximation. Consequently, the side constraints of $\Delta\left(x^{k}\right)$ must be restricted to small values close to zero in order to satisfy the validity of the first order approximation. The definition of the side constraints is usually stated as a centered interval around zero as:

$-\Delta x_{0} \leq \Delta x^{k} \leq \Delta x_{0}$

where $\Delta x_{0}$ is usually defined as:

$\Delta x_{0}=p\left(x_{\max }-x_{\min }\right)$

being $p$ a predefined percentage usually defined in the interval [1\%, 5\%]. This percentage can be slightly modified during the optimization process if needed. In addition, the definition of the side constraints must satisfy:

$x_{\min }-x^{k-1} \leq \Delta x^{k} \leq x_{\max }-x^{k-1}$

to verify the side constraints of the updated design variables of the problem. This definition of side constraints is usually called moving limits in the literature [45-48] since the interval defined by the side constraints of the linearized problem are usually centered around the design variables, which are modified during the optimization problem. The linearized optimization problem, including the side constraints of the design variables, can be stated as:

Minimize $\left.\quad \frac{\partial F}{\partial x}\right|_{x^{k-1}} \Delta x^{k}$

such

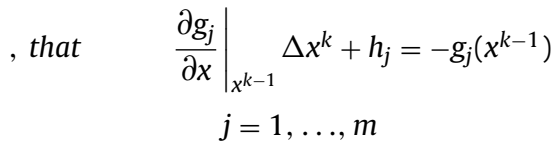

verifying :

$$
\begin{aligned}
& -\Delta x_{0} \leq \Delta x^{k} \leq \Delta x_{0} \\
& x_{\min }-x^{k-1} \leq \Delta x^{k} \leq x_{\max }-x^{k-1}
\end{aligned}
$$

being $F$ the objective function defined according to Section 3.3.2. This linearized optimization problem can be directly solved by applying the Simplex algorithm proposed by G. Dantzig [49]. This algorithm obtains the most adequate value of $\Delta x^{k}$ at each iteration. Thus, the design variables of the problem can be updated according to Section 3.2. The complete iterative optimization process proposed in this paper to solve the optimization of axial turbomachines is usually called in the bibliography Sequential Linear Programming (SLP) [45-48].

\subsubsection{Sensitivity analysis}

The optimization algorithm proposed in Section 5.5 requires the computation of first order derivatives of the objective function and constraints with respect to the design variables of the problem. These derivatives can be computed by numerical methods like finite differences. However, this kind of methods are not advisable if the numerical formulation for the analysis of the problem is available. In this case, the use of analytical methods of derivation is the most effective technique. Numerical methods of derivation are not usually advisable since they introduce sources of error due to the
Table 4

Hypotheses and specifications of inverse and direct design.

\begin{tabular}{ll}
\hline Theoretical parameters & Pump mono-rotor \\
\hline Roughness & $2.0 \mathrm{e}-5(\mathrm{~m})$ \\
Type of vortex & FV-CV-FCV \\
Slack distance between the tip and the hull & $5.0 \mathrm{e}-4(\mathrm{~m})$ \\
Radial equilibrium & Simplified \\
Fairing & Without \\
max thickness of the airfoil rotor & 0.012 \\
Number of sections & 20 \\
Number of the volumetric flow rate & 30 \\
\hline
\end{tabular}

Table 5

Space of search for the designs variables.

\begin{tabular}{llllllllll}
\hline $\begin{array}{l}\text { Side } \\
\text { constraints }\end{array}$ & $Q_{v}$ & $N$ & $R_{i}^{1}$ & $R_{e}^{1}$ & $R_{i}^{2}$ & $R_{e}^{2}$ & $D_{i}$ & $D_{e}$ & $Z$ \\
\hline Min & 0.1 & 1000 & 0.03 & 0.105 & 0.03 & 0.105 & 0.3 & 0.3 & 3 \\
Max & 0.5 & 1900 & 0.100 & 0.150 & 0.100 & 0.150 & 0.7 & 0.7 & 20 \\
Unit & $\mathrm{m}^{3} / \mathrm{s}$ & $\mathrm{rpm}$ & $\mathrm{m}$ & $\mathrm{m}$ & $\mathrm{m}$ & $\mathrm{m}$ & - & - & - \\
\hline
\end{tabular}

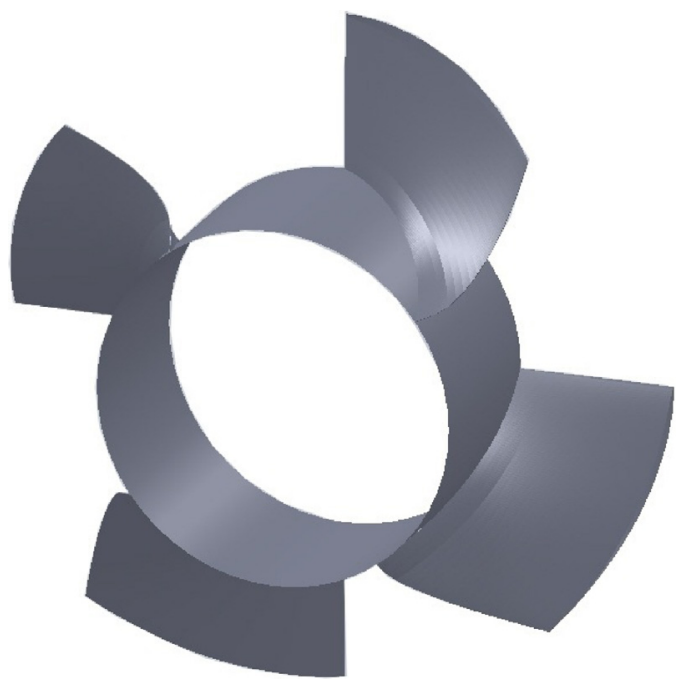

Fig. 6. Reference rotor CAD.

truncation of the approximation series used and due to the properties of the resulting algorithms about round-off errors propagation. In addition, they usually require a large number of analyses of the problem with different input data, which involves in practice considerable computing effort, specially in CPU time. In this paper, the authors have developed analytically the first order derivatives of the objective function proposed in (16) and (17) and the first order derivatives of the constraints proposed in Section 3.4. The authors have also computed analytically the derivatives with respect to all the variables of the problem in order to facilitate the definition of other different optimization problems. Thus, other different optimization problems according to different practical considerations can be easily stated if needed by adequately selecting the design variables and redefining the objective functions and the design constraints.

\section{Optimization of axial pumps}

The axial pump considered as reference in this paper consists of a non shrouded mono rotor, presenting the specifications given in Tables 3 and 4 (Fig. 6). 

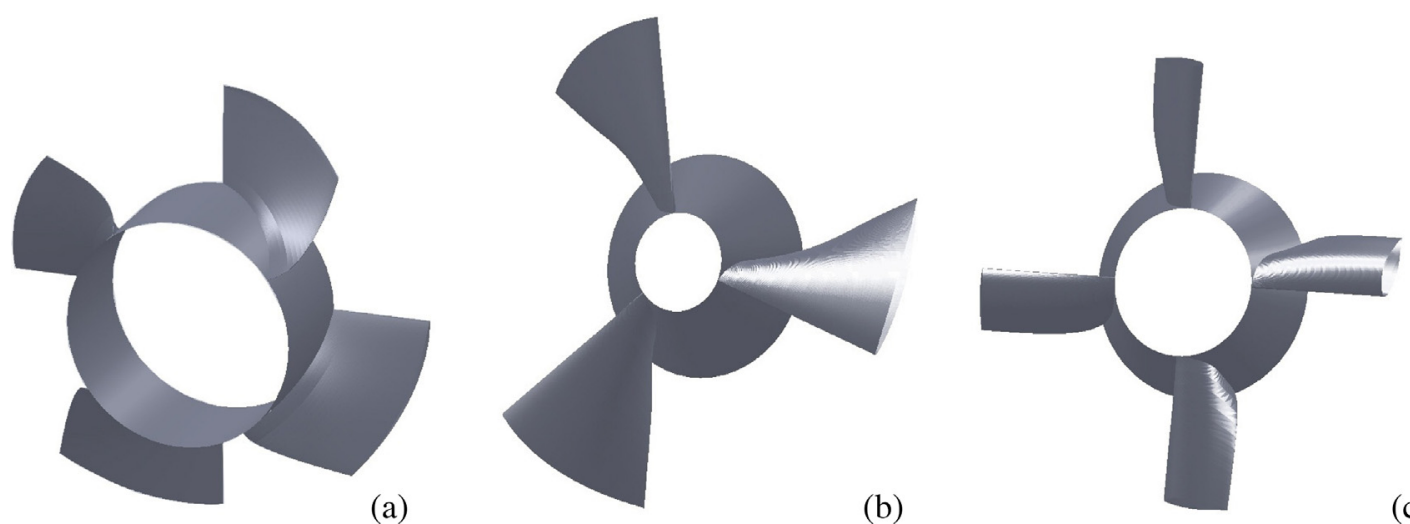

Fig. 7. Case 1-scenario 1: (a) reference rotor CAD, (b) TLBO rotor CAD and (c) SLP rotor CAD.

Table 6

Summary of cases and scenarios optimization studies.

\begin{tabular}{|c|c|c|c|c|c|c|c|c|}
\hline \multirow{2}{*}{$\begin{array}{l}\text { Case } \\
\text { Objective } \\
\text { Scenario }\end{array}$} & \multicolumn{5}{|l|}{$\begin{array}{l}1 \\
\max \left(\eta_{\text {nom }}\right)\end{array}$} & \multicolumn{3}{|c|}{$\max \left(\int_{0.75 Q_{\text {nom }}}^{1.25 Q_{\text {nom }}} \eta\left(Q_{v}\right) d Q_{v}\right)$} \\
\hline & 1 & 2 & 3 & 4 & 5 & 1 & 2 & 3 \\
\hline$N(\mathrm{rpm})$ & 1500 & 1500 & 1500 & 1500 & {$[1000,1900]$} & 1500 & 1500 & {$[1000,1900]$} \\
\hline$Q_{v}\left(\mathrm{~m}^{3} / \mathrm{h}\right)$ & {$[540,1800]$} & {$[540,1800]$} & {$[540,1800]$} & {$[540,1800]$} & 1080 & {$[540,1800]$} & {$[540,1800]$} & 1080 \\
\hline$R_{i}^{1}(\mathrm{~m})$ & {$[0.03,0.1]$} & 0.075 & 0.075 & 0.075 & 0.075 & {$[0.03,0.1]$} & 0.075 & 0.075 \\
\hline$R_{e}^{1}(\mathrm{~m})$ & {$[0.105,0.15]$} & 0.140 & {$[0.126,0.154]$} & 0.140 & {$[0.126,0.154]$} & {$[0.105,0.15]$} & 0.140 & {$[0.126,0.154]$} \\
\hline$R_{i}^{2}(\mathrm{~m})$ & {$[0.03,0.1]$} & 0.075 & 0.075 & 0.075 & 0.075 & {$[0.03,0.1]$} & 0.075 & 0.075 \\
\hline$R_{e}^{\frac{1}{2}}(\mathrm{~m})$ & {$[0.105,0.15]$} & 0.140 & {$[0.126,0.154]$} & 0.140 & {$[0.126,0.154]$} & {$[0.105,0.15]$} & 0.140 & {$[0.126,0.154]$} \\
\hline $\mathrm{Di}$ & {$[0.3,0.7]$} & {$[0.3,0.7]$} & {$[0.3,0.7]$} & {$[0.3,0.7]$} & {$[0.3,0.7]$} & {$[0.3,0.7]$} & {$[0.3,0.7]$} & {$[0.3,0.7]$} \\
\hline De & {$[0.3,0.7]$} & {$[0.3,0.7]$} & {$[0.3,0.7]$} & {$[0.3,0.7]$} & {$[0.3,0.7]$} & {$[0.3,0.7]$} & {$[0.3,0.7]$} & {$[0.3,0.7]$} \\
\hline$Z$ & {$[3,20]$} & {$[3,20]$} & {$[3,20]$} & {$[3,20]$} & {$[3,20]$} & {$[3,20]$} & {$[3,20]$} & {$[3,20]$} \\
\hline Vortex & FV & FV & FV & CV-FCV & FV-CV-FCV & $\mathrm{CV}$ & $\mathrm{CV}$ & $\mathrm{CV}$ \\
\hline
\end{tabular}

Table 7

Results of different methods: case 1-scenario 1.

\begin{tabular}{|c|c|c|c|c|c|c|c|c|c|}
\hline \multirow[t]{2}{*}{$F$} & \multirow[t]{2}{*}{ Reference } & \multicolumn{4}{|l|}{ TLBO } & \multicolumn{4}{|l|}{ CS } \\
\hline & & best & worst & ave & std & best & worst & ave & std \\
\hline Efficiency (\%) & 60.15637 & 70.0543 & 70.0514 & 70.05325 & $8.7591 e-04$ & 70.0474 & 70.0038 & 70.02349 & $1.6508 \mathrm{e}-02$ \\
\hline \multirow[t]{2}{*}{$F$} & PSO & & & & GA & & & & SLP \\
\hline & best & worst & ave & std & best & worst & ave & std & \\
\hline Efficiency (\%) & 70.0546 & 70.0398 & 70.05176 & $5.9939 \mathrm{e}-03$ & 69.4062 & 68.6234 & 69.05351 & $2.9060 \mathrm{e}-01$ & 68.82087 \\
\hline
\end{tabular}

\subsection{Data relative to the algorithmic implementation}

For all presented optimization algorithms, the same variable constraints (side constraints) are considered. The values of these variables are given in Table 5 .

Table 6 shows all the studied cases and scenarios.

The maximum number of function evaluation is fixed as 60,000 for all the algorithms and the run was repeated for 10 times for obtaining the best solution, the worst solution, average (ave) and standard deviation (std). The population size and number of iterations were selected according to the maximum function evaluation allowed. The same computational devices are used as well (CPU Intel Core 2 Duo E5400, 3 GB RAM, 2.9 GHz).

\subsection{Results and discussions}

\subsubsection{Case one: maximization of total efficiency}

6.2.1.1. Scenario one. This first Scenario contains eight decision variables, namely volume flow rate, number of blades, internal and external radius at the inlet and the outlet of the rotor, diffusion factors at the hub and the tip of the rotor, using free vortex strategy. The optimal pump variables with the obtained nominal efficien-

Table 8

Comparison of the optimal solutions: case 1-scenario 1 .

\begin{tabular}{|c|c|c|c|c|c|c|c|c|}
\hline Methods & $Q_{v}\left(\mathrm{~m}^{3} / \mathrm{s}\right)$ & $R_{i}^{1}(\mathrm{~m})$ & $R_{e}^{1}(\mathrm{~m})$ & $R_{i}^{2}(\mathrm{~m})$ & $R_{e}^{2}(\mathrm{~m})$ & $D_{i}$ & $D_{e}$ & $Z$ \\
\hline Reference & 0.3 & 0.075 & 0.14 & 0.075 & 0.14 & 0.68 & 0.32 & 4 \\
\hline TLBO & 0.269755 & 0.0300002 & 0.149148 & 0.0674282 & 0.15 & 0.699999 & 0.347475 & 3 \\
\hline CS & 0.27028 & 0.0302294 & 0.148827 & 0.0677522 & 0.149998 & 0.699927 & 0.343204 & 3 \\
\hline PSO & 0.269788 & 0.0300009 & 0.149043 & 0.0673856 & 0.15 & 0.699992 & 0.346976 & 3 \\
\hline GA & 0.282407 & 0.0399075 & 0.147405 & 0.0731936 & 0.149789 & 0.585075 & 0.33793 & 4 \\
\hline SLP & 0.281981 & 0.0483276 & 0.141835 & 0.0739864 & 0.15 & 0.7 & 0.373599 & 4 \\
\hline
\end{tabular}




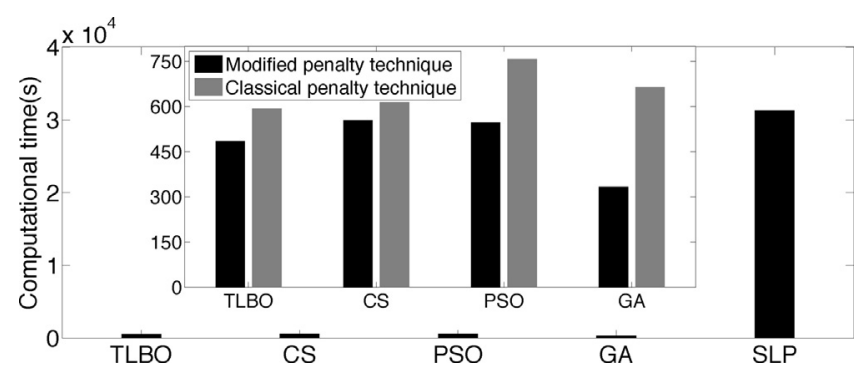

Fig. 8. Comparison of the computational time: case 1-scenario 1.

cies obtained by the proposed optimization methods are given in Tables 7 and 8:

Compared to the results of the reference machine, we record a clear increase of the total efficiency: about $10 \%$ of increase for TLBO, PSO and CS, and about $9 \%$ and $8 \%$ of increase is obtained by GA and SLP respectively.

We notice also that for all the explored methods, the optimal exterior radius, at the inlet and at the outlet of the blade, is close to its maximal value (Fig. 7). The obtained results confirm that the efficiency increases with the decrease of the volume flow rate. Moreover, the diffusion factor at the rotor's hub is raised, but remains as low as possible at the tip.

The average computational time is compared for the various optimization methods and represented in the following histogram (Fig. 8).

It is shown that the SLP algorithm is by far, the heaviest because, besides, this local method, is restricted by a number decision vari-

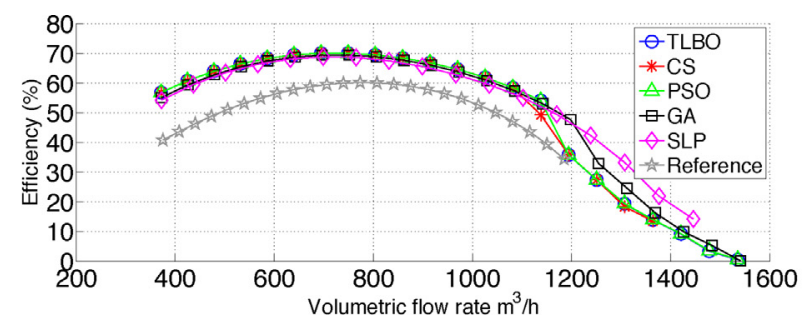

(a) Evolution of total efficiency

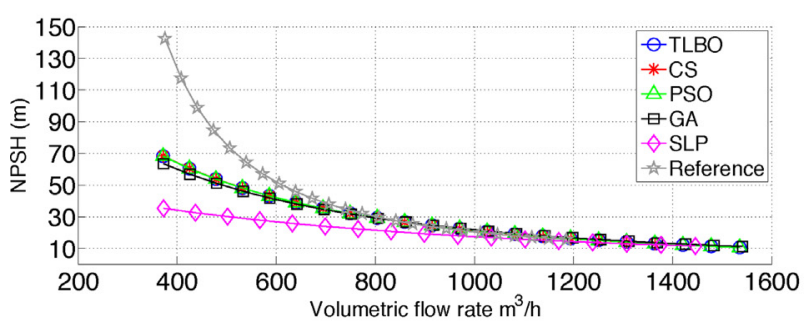

(c) Evolution of the NPSH

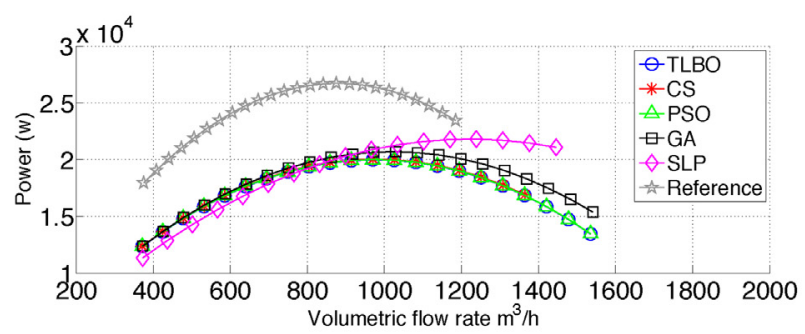

(e) Evolution of the power

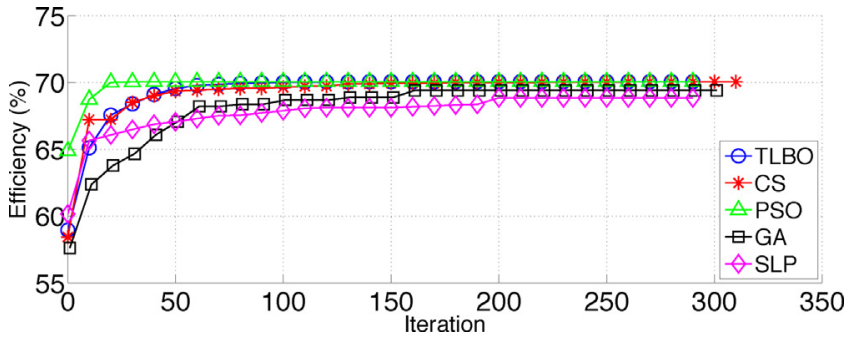

Fig. 9. Comparison of best Fitness's variation with the iterations.

able, which implies the necessity of an iterative calculation for the additional variable. CS and PSO require relatively the same computational time, TLBO records a little decrease of computational time and finally GA that is considered as faster algorithm to the previous algorithms. Modified penalty technique can decrease the computational time with an indeterminate rate because the random nature of used algorithms, after many tests, the value of this rate is between $14 \%$ and $50 \%$.

On the other hand, the fast convergence of the meta heuristics algorithms constitutes another advantage compared to the local methods (Fig. 9). Despite the fact that they are initiated with a random population, contrary to the SLP, which is initiated with a reference solution, we notice that PSO and TLBO converge respectively around 40th and 70th iterations, whereas the GA and CS exceeds half the number of iteration before convergence.

Fig. 25a shows the variation of the efficiency as a function of the volume flow rate. The optimized machines which have the higher

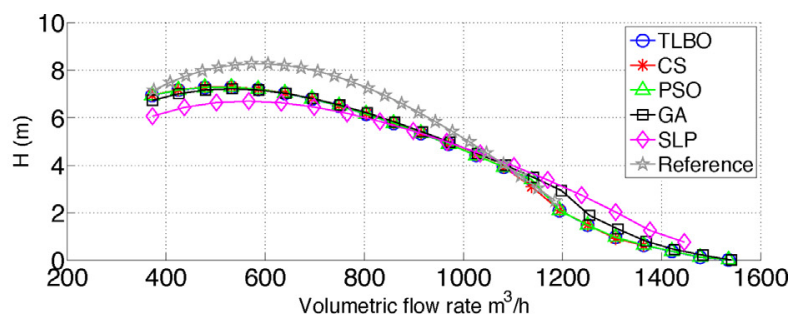

(b) Evolution of the head

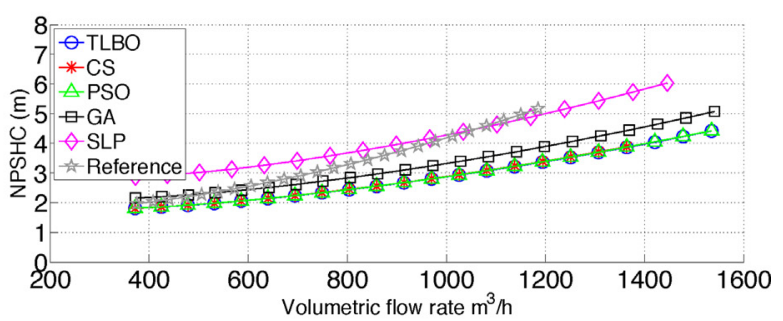

(d) Evolution of the NPSHC

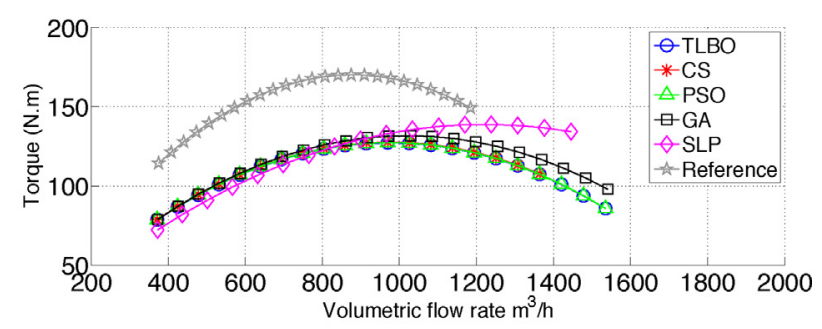

(f) Evolution of the torque

Fig. 10. Case 1-scenario 1: performances. 


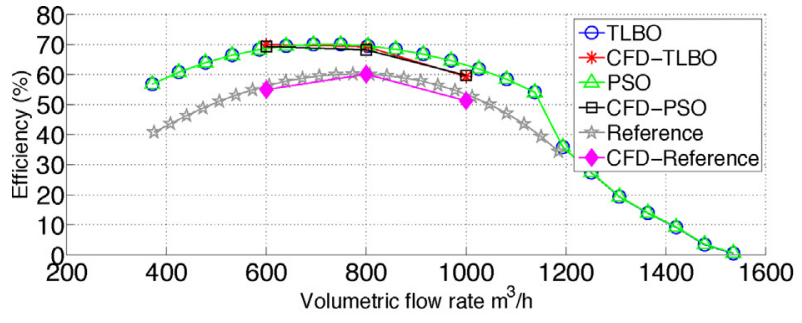

(a) Evolution of total efficiency

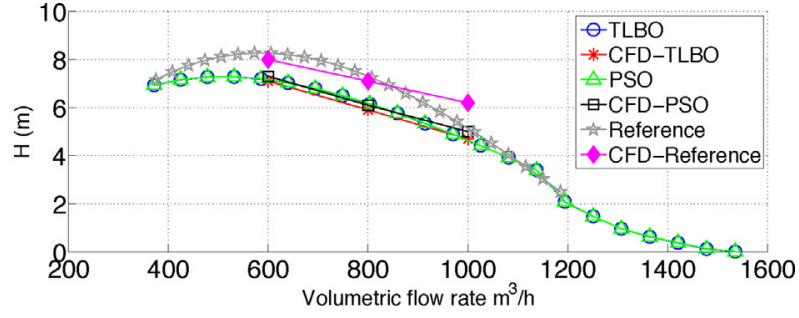

(b) Evolution of the head

Fig. 11. Comparison of the performance prediction of proposed approach with CFD: case 1-scenario 1.

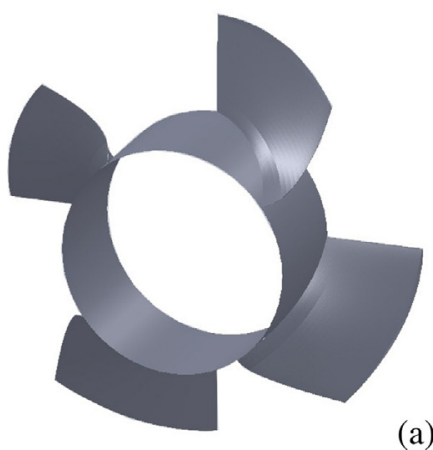

(a)

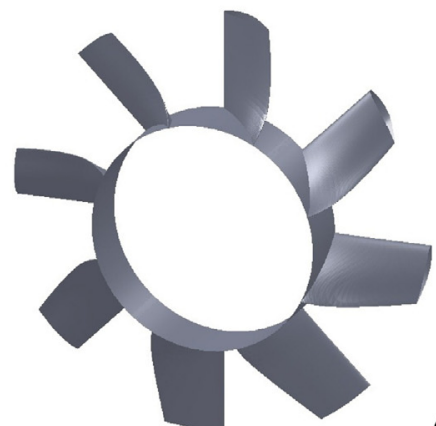

(b)

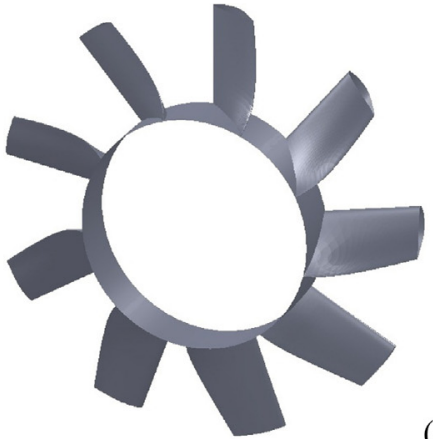

(c)

Fig. 12. Case 1-scenario 2: A) Reference rotor $C A D, B) C S$ rotor $C A D, C)$ GA rotor $C A D$.

Table 9

Results of different methods: case 1-scenario 2.

\begin{tabular}{|c|c|c|c|c|c|c|c|c|}
\hline \multirow[t]{2}{*}{$F$} & \multicolumn{4}{|l|}{ TLBO } & \multicolumn{4}{|l|}{$\mathrm{CS}$} \\
\hline & best & worst & ave & std & best & worst & ave & std \\
\hline Efficiency (\%) & 62.0627 & 62.0619 & 62.06248 & $3.3599 \mathrm{e}-04$ & 62.0626 & 62.0624 & 62.06247 & $6.7495 \mathrm{e}-05$ \\
\hline \multirow[t]{2}{*}{$F$} & \multicolumn{4}{|l|}{ PSO } & \multicolumn{4}{|l|}{ GA } \\
\hline & best & worst & ave & std & best & worst & ave & std \\
\hline Efficiency (\%) & 62.0627 & 62.0621 & 62.06242 & $2.7809 \mathrm{e}-04$ & 62.056 & 61.99784 & 62.0230 & $2.2565 \mathrm{e}-02$ \\
\hline
\end{tabular}

Table 10

Comparison of the optimal solutions: case 1-scenario 2 .

\begin{tabular}{|c|c|c|c|c|}
\hline Methods & $Q_{v}\left(\mathrm{~m}^{3} / \mathrm{s}\right)$ & $D_{i}$ & $D_{e}$ & $Z$ \\
\hline TLBO & 0.284529 & 0.699999 & 0.397594 & 9 \\
\hline CS & 0.284537 & 0.699955 & 0.397678 & 8 \\
\hline PSO & 0.284529 & 0.699999 & 0.397594 & 9 \\
\hline GA & 0.284657 & 0.699962 & 0.398492 & 9 \\
\hline
\end{tabular}

Table 11

Results of different methods: case 1-scenario 3.

\begin{tabular}{|c|c|c|c|c|c|c|c|c|}
\hline \multirow[t]{2}{*}{$F$} & \multicolumn{4}{|l|}{ TLBO } & \multicolumn{4}{|l|}{$\mathrm{CS}$} \\
\hline & best & worst & ave & std & best & worst & ave & std \\
\hline Efficiency (\%) & 69.144 & 69.1395 & 69.13995 & $1.4230 \mathrm{e}-03$ & 69.1393 & 69.1374 & 69.13855 & $7.6485 \mathrm{e}-04$ \\
\hline \multirow[t]{2}{*}{$F$} & \multicolumn{4}{|l|}{ PSO } & \multicolumn{4}{|l|}{ GA } \\
\hline & best & worst & ave & std & best & worst & ave & std \\
\hline Efficiency (\%) & 69.144 & 69.1438 & 69.14398 & $6.3246 e-05$ & 68.5954 & 67.6322 & 68.11708 & $2.9908 \mathrm{e}-01$ \\
\hline
\end{tabular}

nominal efficiency present also a higher off-design efficiency compared to the reference pump.

In this paper, we are exclusively interested on the improvement of the total nominal efficiency, the cavitation criterion was not considered. In Fig. 10c and d, it is noticed that for all machines, the required net pressure suction head (NPSHC) is inferior to the NPSH on all the range of volume flow rate. i.e. the cavitation criterion is respected.

Another positive point of optimized pumps, its consumed power is less than reference pump for all volumetric flow rate range (Fig. 10e and f). 
62.5

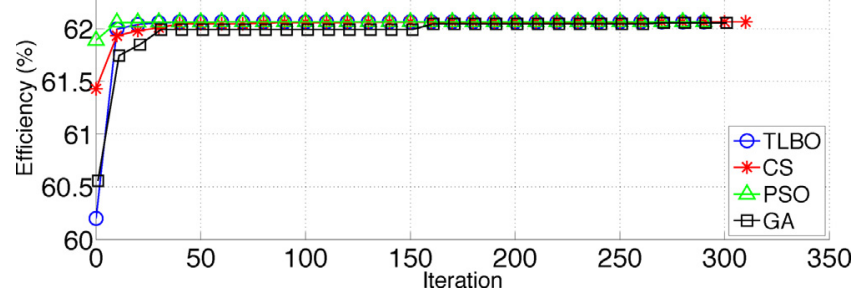

Fig. 13. Comparison of best fitness's variation with the iterations: case 1-scenario

The CFD method using Ansys-Fluent is used to predict the performance of two first best optimized machines (i.e. PSO and TLBO) as well as the reference machine. A steady flow is imposed using Moving Reference frame with periodic boundary condition. The turbulence model $K-\omega S S T$ is chosen for solving three-dimensional Reynolds-averaged Navier-Stokes equations. For the design point neighborhood the results of CFD are very acceptable, is so closed to the analysis of the proposed approaches (Fig. 11). For the reference machine, the average relative error is about $2.11 \%$ and $13.60 \%$ for efficiency and head respectively. For TLBO's machine, the average relative error is about $2.86 \%$ and $1.96 \%$ for efficiency and head respectively. For the PSO's machine, the average relative error is about $2.96 \%$ and $3.45 \%$ for efficiency and head respectively.

6.2.1.2. Scenario two. In the second scenario, the inlet and outlet radius are kept constant. For the following cases and starting from this scenario, the SLP method is not explored.

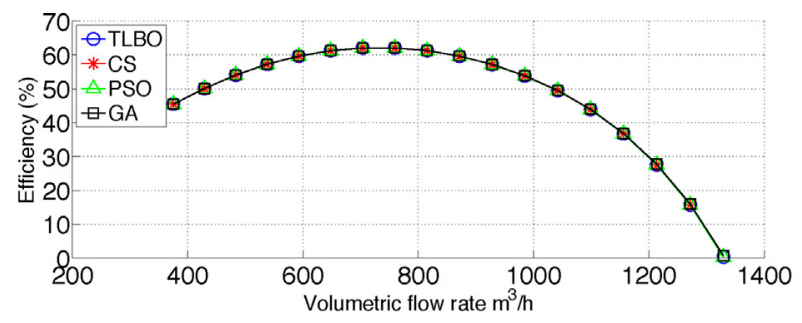

(a) Evolution of total efficiency

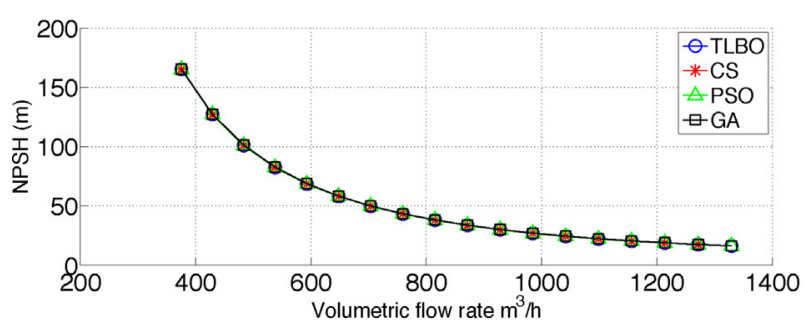

(c) Evolution of the NPSH

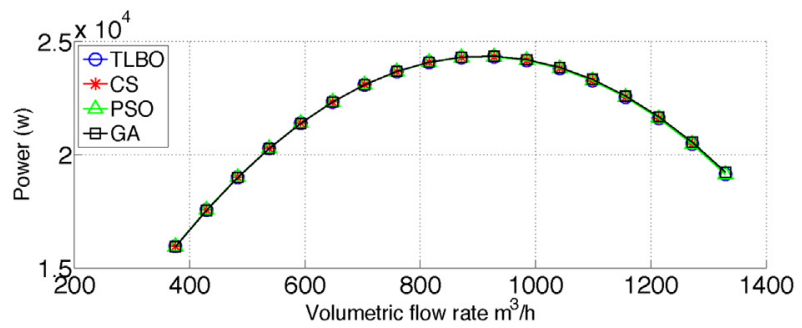

(e) Evolution of the power
Table 12

Comparison of the optimal solutions: case 1-scenario 3.

\begin{tabular}{lllllll}
\hline Methods & $Q_{v}\left(\mathrm{~m}^{3} / \mathrm{s}\right)$ & $R_{e}^{1}(\mathrm{~m})$ & $R_{e}^{2}(\mathrm{~m})$ & $D_{i}$ & $D_{e}$ & $Z$ \\
\hline TLBO & 0.269755 & 0.153302 & 0.154 & 0.7 & 0.492199 & 11 \\
CS & 0.250453 & 0.153287 & 0.154 & 0.699995 & 0.491273 & 11 \\
PSO & 0.282407 & 0.153298 & 0.154 & 07 & 0.492113 & 11 \\
GA & 0.258843 & 0.150389 & 0.152934 & 0.6992 & 0.510518 & 11 \\
\hline
\end{tabular}

Tables 9 and 10 and Fig. 13 show the convergence of the algorithms to nearly the same solution, with an increase in total efficiency by almost $2 \%$ relative to the reference machine. The performance curves presented in Fig. 14 show that the obtained machines are similar. Further, Fig. 14e and f shows that the power consumed by these machines is less than the power consumed by the reference machine. Figs. 14c and d shows that the cavitation criterion is satisfied. Finally, the geometries are relevant in terms of design as shown in Fig. 12. Observe the difference in the rotor axial distance between the optimized machines where the constraints are respected and the reference machine where they are not.

6.2.1.3. Scenario three. In the third scenario, only the hub radius are kept constant, the external radius vary in a range of $-10 \%$ to $+10 \%$ of the external radius of the reference machine.

Tables 11 and 12 and Fig. 16 show that the rate of increase in total efficiency is about $9 \%$ for the TLBO, PSO and CS, and less then $9 \%$ for GA. Notice that the radius tend to their maximum value in the search space as well as the diffusion factor at the hub. Notice also that there is also an arguably high number of blades compared to the other scenarios.

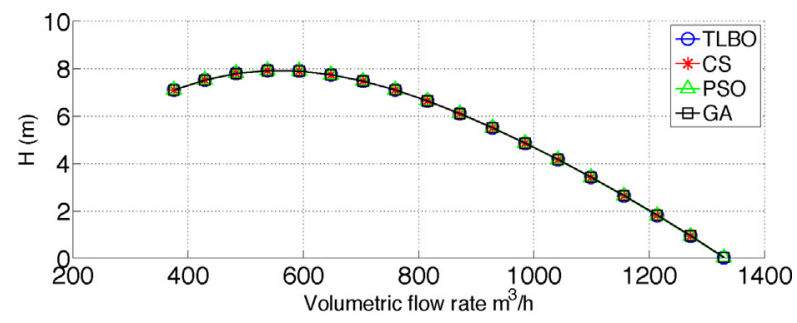

(b) Evolution of the head

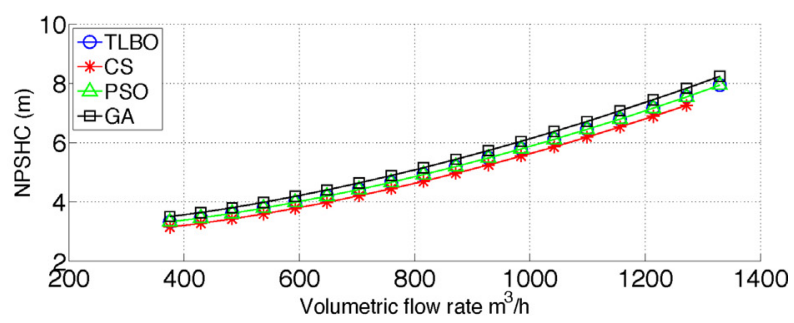

(d) Evolution of the NPSHC

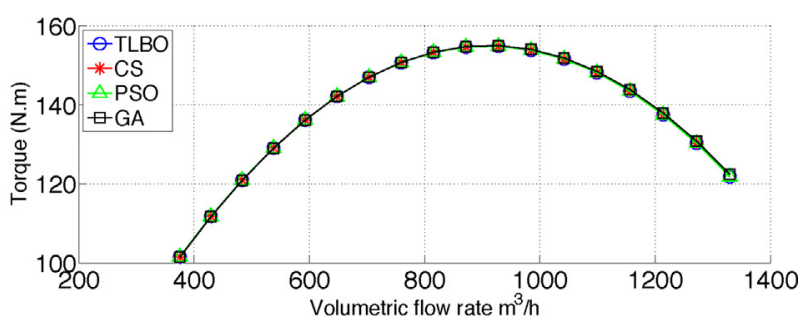

(f) Evolution of the torque

Fig. 14. Case 1-scenario 2: performances. 


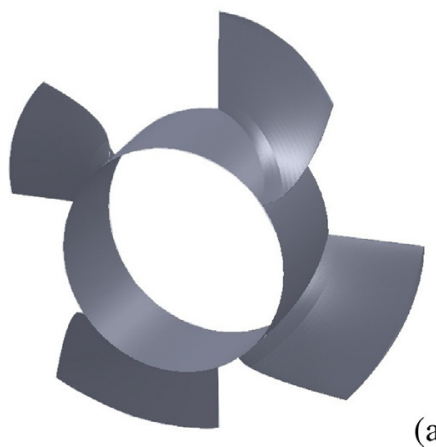

(a) (b)

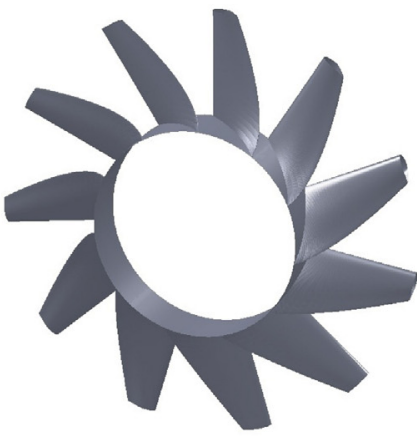

(c)

Fig. 15. Case 1-scenario 3: (a) reference rotor CAD, (b) PSO rotor CAD and (c) GA rotor CAD.

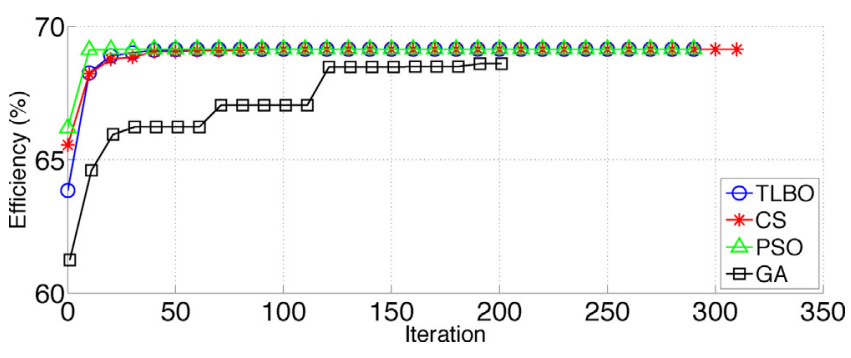

Fig. 16. Comparison of best fitness's variation with the iterations: case 1-scenario

Except for GA, Fig. 17 shows homogeneity and coincidence of the efficiency curve (Fig. 17a), the head (Fig. 17b), NPSH and NPSHC

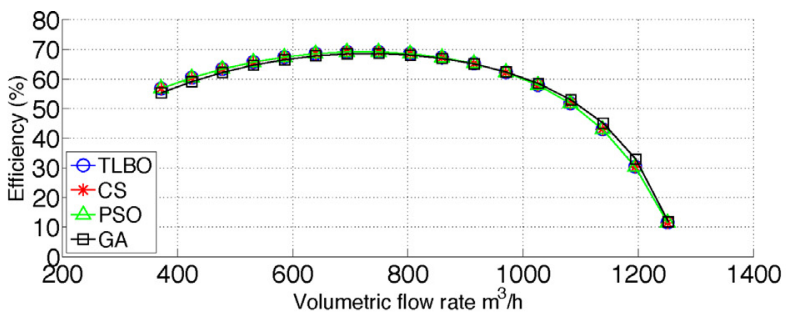

(a) Evolution of total efficiency

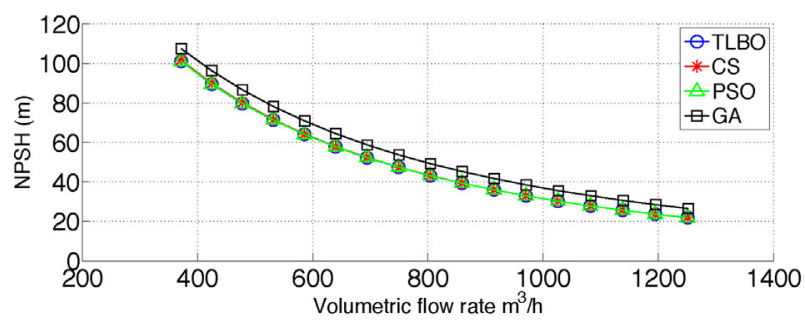

(c) Evolution of the NPSH

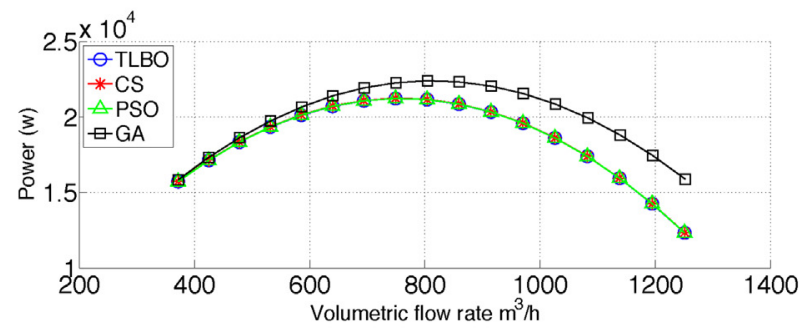

(e) Evolution of the power
(Fig. 17d and c respectively), which verifies the cavitation criterion. The curves of torque and power present a coincidence between the PSO, CS and TLBO as shown in Fig. $17 \mathrm{f}$ and e. Finally for all algorithms, the power is always lower than that of the reference machine. The geometries given by PSO and GA are comparable as shown in Fig. 15.

6.2.1.4. Scenario four. The fourth scenario is similar to the second one concerning the decision variables except that optimization is done for two types of vortex, namely constant and forced (Fig. 18).

Tables 13 and 14 and Fig. 19 show that the use of forced and constant vortex can slightly improve the performance, particularly the forced vortex. For the diffusion factor, the results show that the obtained machine can operate with a marginally high values, either

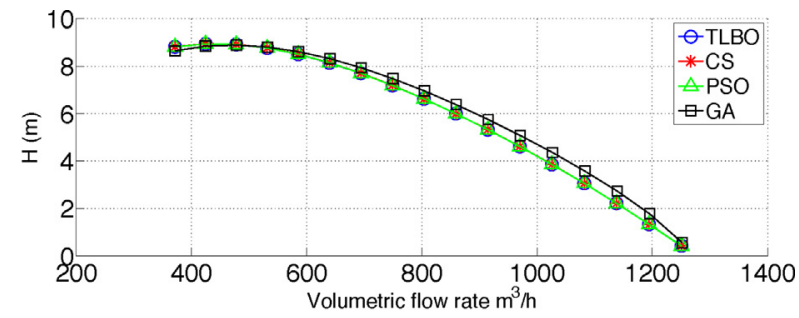

(b) Evolution of the head

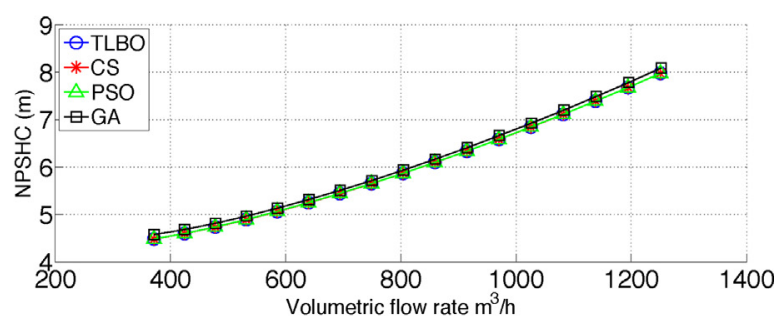

(d) Evolution of the NPSHC

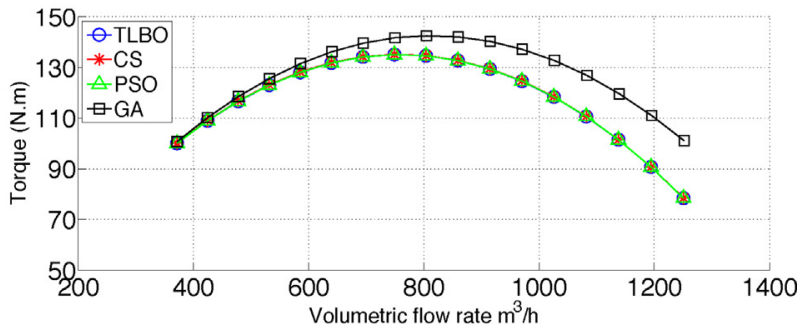

(f) Evolution of the torque

Fig. 17. Case 1-scenario 3: performances. 


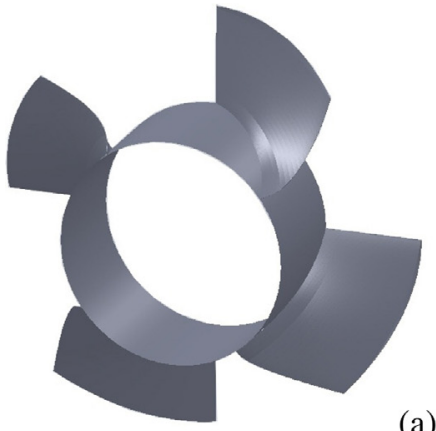

(a)

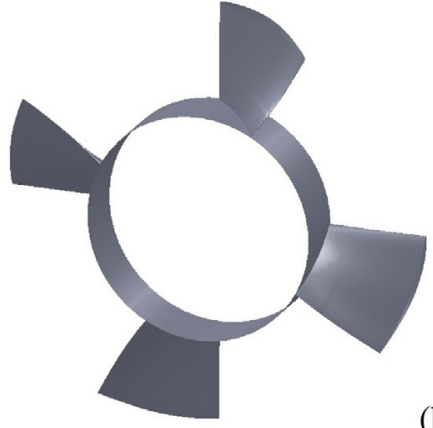

(b)

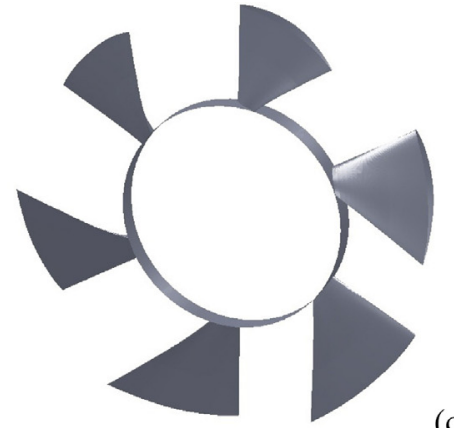

(c)

Fig. 18. Case 1-scenario 4: (a) reference rotor CAD, (b) CS-CV rotor CAD and (c) CS-FCV rotor CAD.

Table 13

Results of different methods: case 1-scenario 4.

\begin{tabular}{|c|c|c|c|c|c|c|c|c|c|}
\hline \multirow[t]{2}{*}{$F$} & & \multicolumn{4}{|l|}{ TLBO } & \multicolumn{4}{|l|}{ CS } \\
\hline & & best & worst & ave & std & best & worst & ave & std \\
\hline \multirow[t]{2}{*}{ Efficiency (\%) } & $\mathrm{CV}$ & 63.3594 & 63.3527 & 63.35793 & $1.9282 \mathrm{e}-03$ & 63.3595 & 63.3524 & 63.3565 & $3.4351 \mathrm{e}-03$ \\
\hline & FCV & 63.6206 & 63.614 & 63.61993 & $2.0838 \mathrm{e}-03$ & 63.6201 & 63.614 & 63.61604 & $2.7048 \mathrm{e}-03$ \\
\hline \multirow[t]{2}{*}{$F$} & & \multicolumn{4}{|l|}{ PSO } & \multicolumn{4}{|l|}{ GA } \\
\hline & & best & worst & ave & std & best & worst & ave & std \\
\hline \multirow[t]{2}{*}{ Efficiency (\%) } & $\mathrm{CV}$ & 63.3594 & 63.3527 & 63.35739 & $2.5049 \mathrm{e}-03$ & 63.3595 & 63.3149 & 63.33529 & $1.1766 \mathrm{e}-02$ \\
\hline & FCV & 63.6206 & 63.614 & 63.61925 & $2.3206 \mathrm{e}-03$ & 63.6132 & 63.6108 & 63.61202 & $8.5219 \mathrm{e}-04$ \\
\hline
\end{tabular}

Table 14

Comparison of the optimal solutions: case 1-scenario 4

\begin{tabular}{llllll}
\hline Methods & & $Q_{v}\left(\mathrm{~m}^{3} / \mathrm{s}\right)$ & $D_{i}$ & $D_{e}$ & $Z$ \\
\hline TLBO & CV & 0.294284 & 0.698088 & 0.516567 & 11 \\
& FCV & 0.296991 & 0.55635 & 0.684712 & 9 \\
CS & CV & 0.294174 & 0.698246 & 0.515346 & 4 \\
& FCV & 0.296967 & 0.556322 & 0.684121 & 6 \\
PSO & CV & 0.29425 & 0.69814 & 0.51619 & 11 \\
& FCV & 0.297017 & 0.556317 & 0.685256 & 9 \\
GA & CV & 0.294325 & 0.699532 & 0.51403 & 4 \\
& FCV & 0.297048 & 0.541793 & 0.690215 & 4 \\
\hline
\end{tabular}

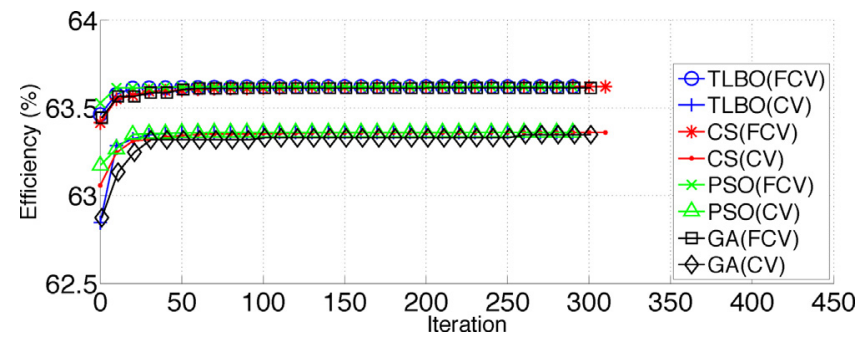

Fig. 19. Comparison of best fitness's variation with the iterations for different vortex: case 1 -scenario 4

with forced or constant vortex. Fig. 20a illustrates the improvement in the efficiency between the different types of vortex. Notice that the optimized machines provide a little different charges depending on the vortex type, also the forced vortex and constant vortex gives the highest and lowest heads respectively than the free vortex, see Fig. 20b.

Fig. 20c and d shows that the cavitation criterion is respected. Fig. 20e and f confirms this observation for the power consumption. In the other side, at nominal point, all the optimized machines present less power compared to the reference machine for all considered types of vortex.
6.2.1.5. Scenario five. In the fifth scenario, the flow rate is kept constant and the rotational speed variable and the hub radius are also fixed. The outer radius is variables within a range of $-10 \%$ to $10 \%$ of the tip radius of the reference machine. The machines are optimized for the three types of vortex (free, forced and constant). In this scenario, presented in Table 15 and Fig. 21, the efficiency can be improved by $14 \%$ and $13 \%$. Notice that the machines operate with the maximum rotational speed Table 16. Moreover, all tip radius and are close to same solution that is the maximum allowed value. In this scenario, the relevant solutions are present comparable performances as shown in Fig. 22.

\subsubsection{Case two: maximization the operation area under the efficiency curve}

In this case we propose a new objective function for the design of a turbomachine, it consists on maximizing the area under the performance curves, in our case it is the efficiency curves. A numerical integration was used on a flow range of $-25 \%$ to $25 \%$ of the nominal rate. A constraint is added to the head, which is the head variation within this range, and that should not exceed $15 \%$ of the head of the nominal point. Only constant vortex has been considered in this case.

6.2.2.1. Scenario one. In this scenario all the parameters are considered variables. Tables 17 and 18 and Fig. 23 show an increase of this area by $60.590 \%$ and $60.935 \%$ with TLBO and PSO respectively and by $60.558 \%$ and $59.963 \%$ with CS and GA respectively. It is found that this objective can increase the design efficiency. Fig. 24a shows the difference in the neighborhood area of nominal flow rate between the reference machine and the optimized machine. The constraint of variation limitation of the head is well respected as presented in Fig. 24b. Fig. 24e and f shows that this objective, in which the efficiency variation range is wider, causes higher power consumption, and on a proportionate basis with the flow rate.

Using the same CFD condition of the first case, in this second case and for the design point neighborhood of TLBO's machine, the 


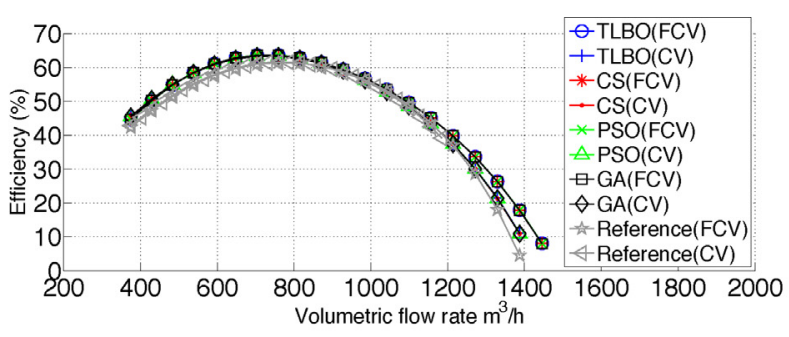

(a) Evolution of total efficiency

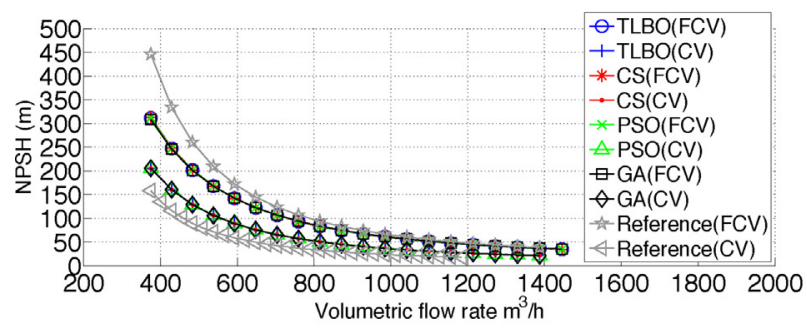

(c) Evolution of the NPSH

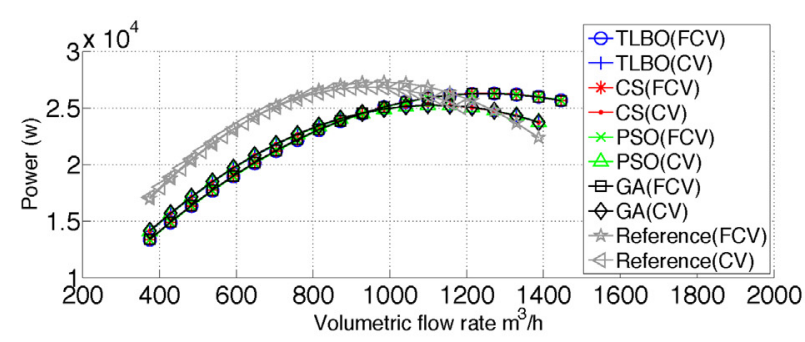

(e) Evolution of the power

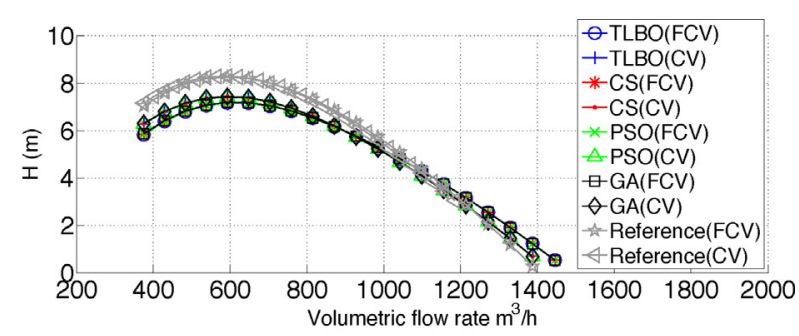

(b) Evolution of the head

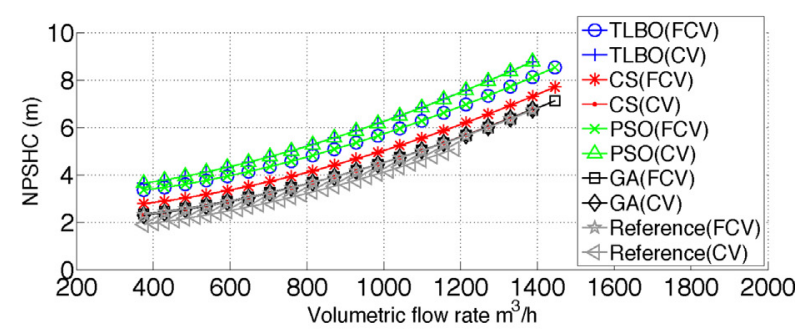

(d) Evolution of the NPSHC

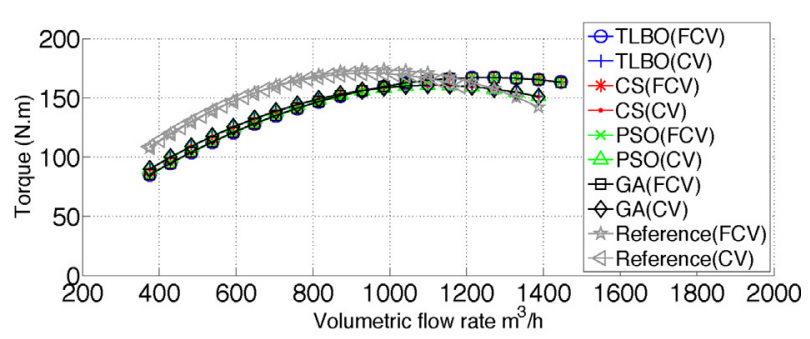

(f) Evolution of the torque

Fig. 20. Case 1-scenario 4: performances.

Table 15

Results of different methods: case 1-scenario 5.

\begin{tabular}{|c|c|c|c|c|c|c|c|c|c|}
\hline \multirow[t]{2}{*}{$F$} & & \multicolumn{4}{|l|}{ TLBO } & \multicolumn{4}{|l|}{ CS } \\
\hline & & best & worst & ave & std & best & worst & ave & std \\
\hline \multirow[t]{3}{*}{ Efficiency (\%) } & FV & 73.4246 & 73.4246 & 73.4246 & $1.4980 \mathrm{e}-14$ & 73.4246 & 73.4246 & 73.4246 & $1.4980 \mathrm{e}-14$ \\
\hline & $\mathrm{CV}$ & 74.6334 & 74.6334 & 74.6334 & $1.4980 \mathrm{e}-14$ & 74.6334 & 74.6334 & 74.6334 & $1.4980 \mathrm{e}-14$ \\
\hline & FCV & 74.734 & 74.734 & 74.734 & $1.4980 \mathrm{e}-14$ & 74.734 & 74.734 & 74.734 & $1.4980 \mathrm{e}-14$ \\
\hline
\end{tabular}

\begin{tabular}{|c|c|c|c|c|c|c|c|c|c|}
\hline \multirow[t]{2}{*}{$F$} & & \multicolumn{4}{|l|}{ PSO } & \multicolumn{4}{|l|}{ GA } \\
\hline & & best & worst & ave & std & best & worst & ave & std \\
\hline \multirow[t]{3}{*}{ Efficiency (\%) } & FV & 73.4246 & 73.4246 & 73.4246 & $1.4980 \mathrm{e}-14$ & 73.3955 & 73.3018 & 73.35107 & $3.0256 e-02$ \\
\hline & $\mathrm{CV}$ & 74.6334 & 73.5961 & 74.52967 & $3.2802 \mathrm{e}-01$ & 74.63295 & 74.6177 & 74.6247 & $5.7797 e-03$ \\
\hline & FCV & 74.734 & 73.8391 & 74.64451 & $2.8299 \mathrm{e}-01$ & 74.7305 & 74.7157 & 74.72102 & $4.1635 e-03$ \\
\hline
\end{tabular}

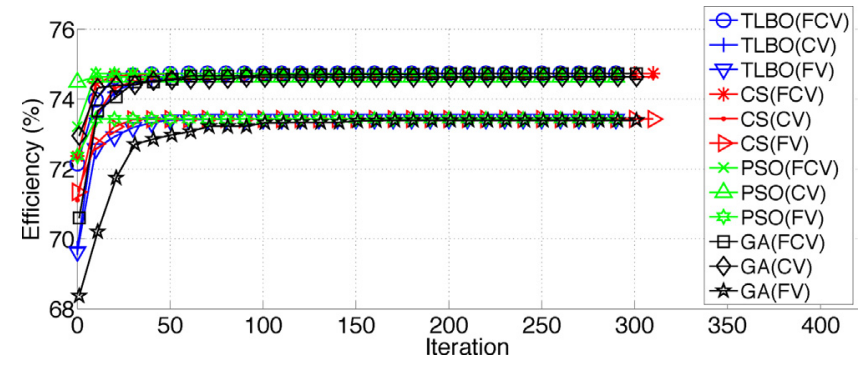

Fig. 21. Comparison of best fitness's variation with the iterations for different vortex: case 1 -scenario 5 . results of CFD are acceptable, the average relative error is about $1.93 \%$ and $2.274 \%$ for efficiency and head respectively.

6.2.2.2. Scenario two. This scenario has the same considerations with the first scenario except that all the radius are kept constant (Fig. 26). Table 19 and Fig. 27 show that the efficiency variation range may be extended even with a small variation about an increase of $7.66 \%$. Almost all algorithms converge to the same solution and all the obtained solutions are geometrically relevant (Table 20). As for the previous scenario, Fig. 28 shows that the maximization of the efficiency range does not always lead to a maximum nominal efficiency. 


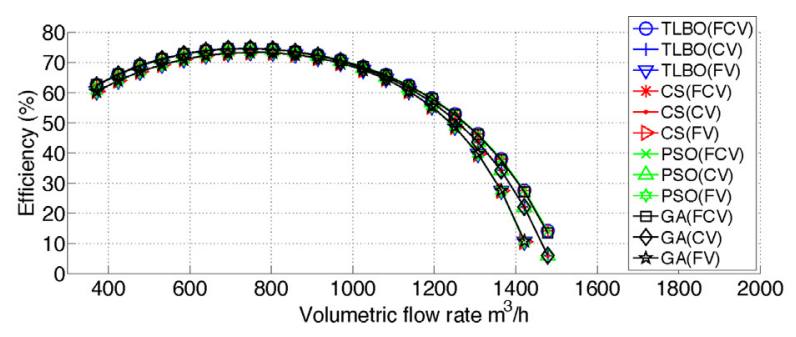

(a) Evolution of total efficiency

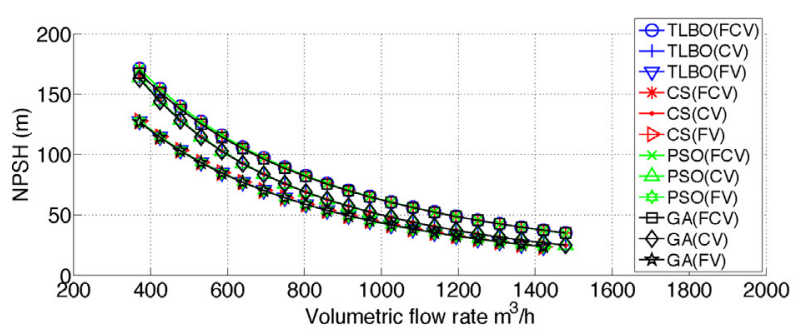

(c) Evolution of the NPSH

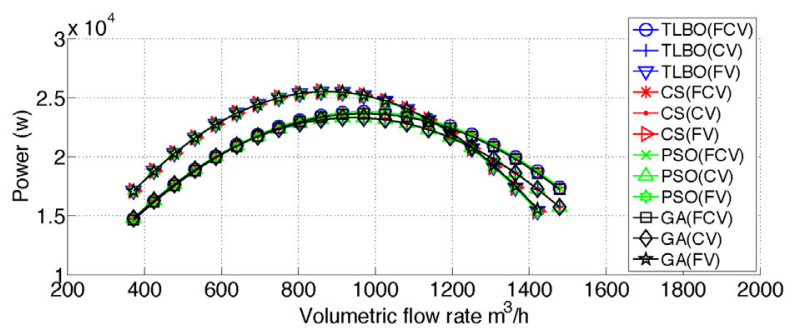

(e) Evolution of the power

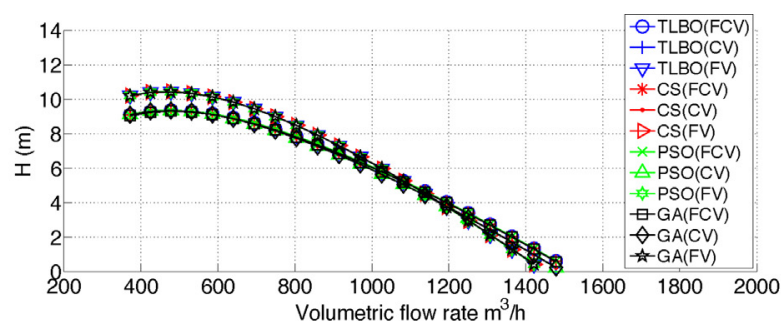

(b) Evolution of the head

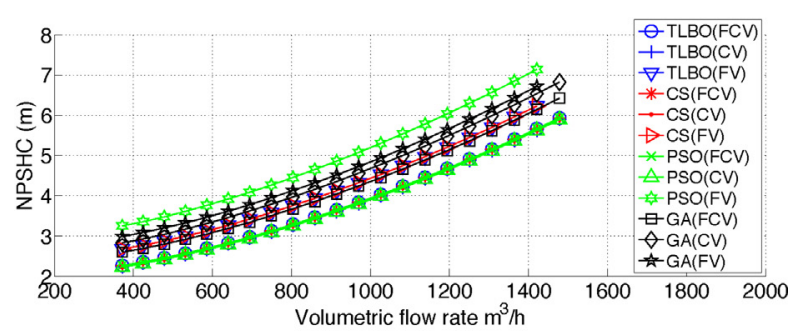

(d) Evolution of the NPSHC

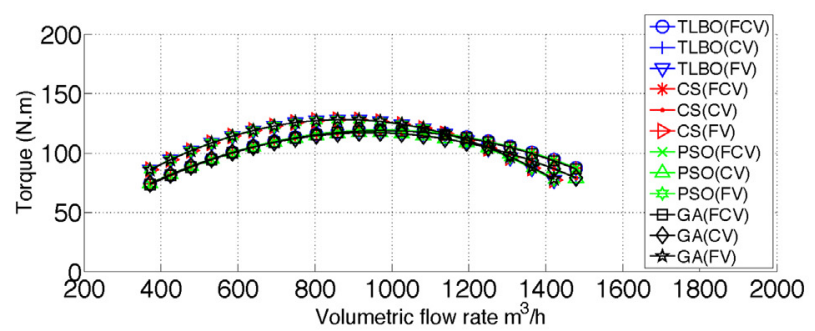

(f) Evolution of the torque

Fig. 22. Case 1-scenario 5: performances.

Table 16

Comparison of the optimal solutions: case 1-scenario 5 .

\begin{tabular}{|c|c|c|c|c|c|c|c|}
\hline Methods & & $N(\mathrm{rpm})$ & $R_{e}^{1}(\mathrm{~m})$ & $R_{e}^{2}(\mathrm{~m})$ & $D_{i}$ & $D_{e}$ & $Z$ \\
\hline \multirow[t]{3}{*}{ TLBO } & FV & 1900 & 0.154 & 0.154 & 0.7 & 0.3 & 4 \\
\hline & CV & 1900 & 0.154 & 0.154 & 0.684502 & 0.371043 & 3 \\
\hline & FCV & 1900 & 0.154 & 0.154 & 0.426198 & 0.513194 & 3 \\
\hline \multirow[t]{3}{*}{ CS } & FV & 1900 & 0.154 & 0.154 & 0.7 & 0.3 & 5 \\
\hline & $\mathrm{CV}$ & 1900 & 0.154 & 0.154 & 0.684502 & 0.371043 & 3 \\
\hline & FCV & 1900 & 0.154 & 0.154 & 0.426198 & 0.513194 & 3 \\
\hline \multirow[t]{3}{*}{ PSO } & FV & 1900 & 0.154 & 0.154 & 0.7 & 0.3 & 7 \\
\hline & CV & 1900 & 0.154 & 0.154 & 0.684502 & 0.371043 & 3 \\
\hline & FCV & 1900 & 0.154 & 0.154 & 0.426198 & 0.513194 & 3 \\
\hline \multirow[t]{3}{*}{ GA } & FV & 1900 & 0.153843 & 0.153921 & 0.699947 & 0.30039 & 5 \\
\hline & $\mathrm{CV}$ & 1900 & 0.153999 & 0.153996 & 0.683323 & 0.371847 & 5 \\
\hline & FCV & 1900 & 0.153843 & 0.153921 & 0.699947 & 0.30039 & 5 \\
\hline
\end{tabular}

Table 17

Results of different methods: case 2-scenario 1.

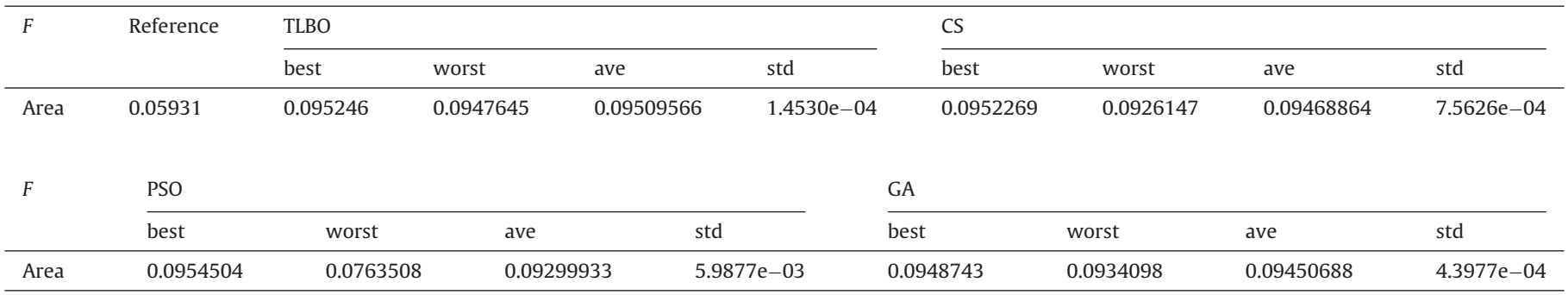


Table 18

Comparison of the optimal solutions: case 2-scenario 1.

\begin{tabular}{|c|c|c|c|c|c|c|c|c|}
\hline Methods & $Q_{v}\left(\mathrm{~m}^{3} / \mathrm{s}\right)$ & $R_{i}^{1}(\mathrm{~m})$ & $R_{e}^{1}(\mathrm{~m})$ & $R_{i}^{2}(\mathrm{~m})$ & $R_{e}^{2}(\mathrm{~m})$ & $D_{i}$ & $D_{e}$ & $Z$ \\
\hline Reference & 0.3 & 0.075 & 0.14 & 0.075 & 0.14 & 0.68 & 0.32 & 4 \\
\hline TLBO & 0.441313 & 0.030153 & 0.140272 & 0.0348952 & 0.149991 & 0.691658 & 0.593822 & 5 \\
\hline CS & 0.442526 & 0.030381 & 0.144615 & 0.0351488 & 0.149994 & 0.646998 & 0.638884 & 4 \\
\hline PSO & 0.441199 & 0.03 & 0.145582 & 0.0364082 & 0.15 & 0.699095 & 0.6172 & 4 \\
\hline GA & 0.445684 & 0.0317479 & 0.144948 & 0.0376835 & 0.149819 & 0.638295 & 0.621847 & 6 \\
\hline
\end{tabular}

Table 19

Results of different methods: case 2-scenario 2.

\begin{tabular}{|c|c|c|c|c|c|c|c|c|}
\hline \multirow[t]{2}{*}{$F$} & \multicolumn{4}{|l|}{ TLBO } & \multicolumn{4}{|l|}{ CS } \\
\hline & best & worst & ave & std & best & worst & ave & std \\
\hline Area & 0.0638551 & 0.0638526 & 0.06385324 & $9.9017 e-07$ & 0.0638527 & 0.0638386 & 0.06384904 & $4.1358 \mathrm{e}-06$ \\
\hline \multirow[t]{2}{*}{$F$} & \multicolumn{4}{|l|}{ PSO } & \multicolumn{4}{|l|}{ GA } \\
\hline & best & worst & ave & std & best & worst & ave & std \\
\hline Area & 0.0638551 & 0.0638471 & 0.06385336 & $2.3477 \mathrm{e}-06$ & 0.0638449 & 0.0637933 & 0.06382547 & $1.8558 \mathrm{e}-05$ \\
\hline
\end{tabular}

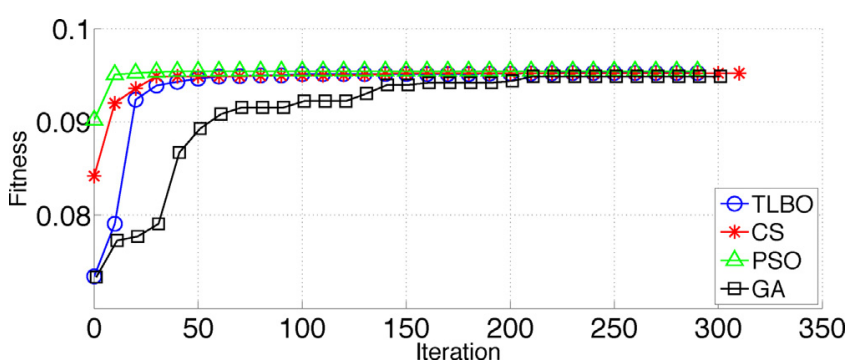

Fig. 23. Comparison of best Fitness's variation with the iterations: case 2-scenario 1.

Table 20

Comparison of the optimal solutions: case 2-scenario 2.

\begin{tabular}{lllll}
\hline Methods & $Q_{v}\left(\mathrm{~m}^{3} / \mathrm{s}\right)$ & $D_{i}$ & $D_{e}$ & $Z$ \\
\hline TLBO & 0.426242 & 0.671216 & 0.478967 & 9 \\
CS & 0.426466 & 0.657295 & 0.480458 & 8 \\
PSO & 0.42624 & 0.671392 & 0.478946 & 9 \\
GA & 0.426003 & 0.650969 & 0.486162 & 4
\end{tabular}

6.2.2.3. Scenario three. In this scenario only the flow and the hub radius are kept constant. The tip radius varies within a range of $-10 \%$ to $+10 \%$ of the tip radius of the reference machine. Tables 21 and 22 and Fig. 29 show that the algorithms converge to the approximate solution, and the rate of increase is lower than the first scenario and higher than the second one (9.56\%). Concerning the performance, a high rotational speed can increase the nominal efficiency and the area with a lower power consumption, as shown in Fig. 30.

\subsection{Recapitulation of algorithms behavior}

TLBO, CS and PSO record a higher and approximate solution, and the error between each other is between $10^{-4}$ and $10^{-6}$. Compared with previous methods, almost for all cases, GA was recorded having the worst behavior whether of the way of the convergence or the solution (namely with more decision variable) may be because it needs more function evaluations or the parameters of this algorithm do not well adjusted which is considered as an inconvenient, because there is no rule to determine this parameter, they are still strongly linked to the optimization problem. SLP is limited with hard problem like that considered in this paper because of its implementation complexity and its local solution.
Table 23 summarizes the performance ranking of the solutions found and the stability of the algorithms used. For each scenario, the algorithms were classified from 1 to 4 according to their best fitness, worst fitness, average fitness and standard deviation. After that, the sum of the rank was computed for each algorithm and for each statistical parameter in order to find the nearness of the algorithm to the ideal one (that presents the first rank for all scenarios). Finally, the average rank of each method was calculated to define the overall rank. Based on this, TLBO present the best stability (the nearest one), followed by CS and PSO, and finally GA. We note that the ranking is correspond controlling parameters of the algorithm which the TLBO does not need any parameters flowing by CS that has fewer parameters (only one, the empty nest rate Pa), in the third rank we found PSO that uses three parameters (inertia weight, cognitive and social parameters) and final GA which works with more than three parameters (crossover rate and mutation rate, scale, ratio and shrink).

\subsection{Comparison of the proposed approach with some existing machines}

To demonstrate the effectiveness of the proposed framework, some baseline pumps (pump A [50], pump B and pump C [25]) from literature are compared with optimized machines using TLBO under same specifications. The strategy of comparison is based on two scenarios, the first (S1) is to fix the maximum outer radius constraint equal to the value of the reference pump's radius, the second one (S2), is to extend maximum side constraint radius with $+10 \%$ of the reference value. For both scenarios and for pump A, the objectives are the maximization of nominal total efficiency (main formulation in pervious case 1 (C1)) and area of total efficiency-flow rate (main formulation in pervious case $2(\mathrm{C} 2)$ ). The maximization of the nominal hydraulic efficiency is considered for pump $B$ and $C$.

Table 24 shows the optimal geometrical parameters and their operating conditions compared with the reference pump A. In the first objective and for scenario S1, the improvement of nominal efficiency is very small (about $0.01 \%$ ) but the respecting of the head in point of design is clear. With the second scenario, the difference between optimized pump and the reference one is about 7\%. In addition, the optimized machines have a good distribution of the performance curves, as shown in Fig. 31, the curves quality is recorded for all flow rate range with the efficiency, and for neighborhoods design point flow rate with the head curve. For the second objective, for both scenarios the difference between the optimized pumps area and reference pump area is clear, it is about $8 \%$ for first 


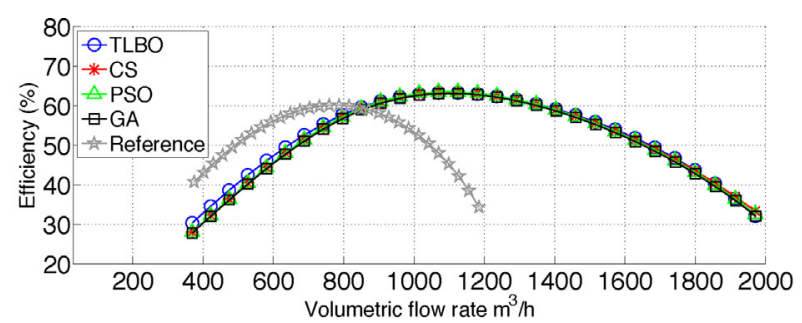

(a) Evolution of total efficiency

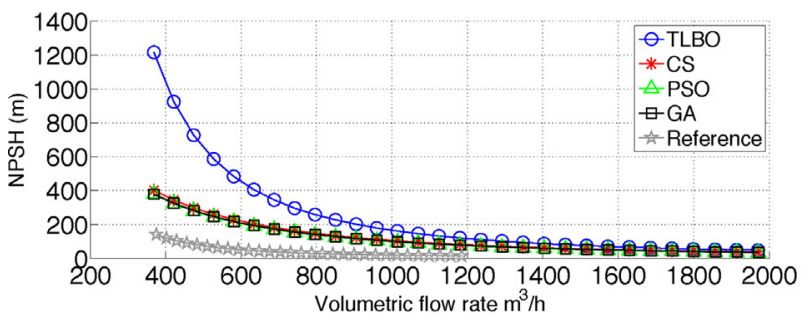

(c) Evolution of the NPSH

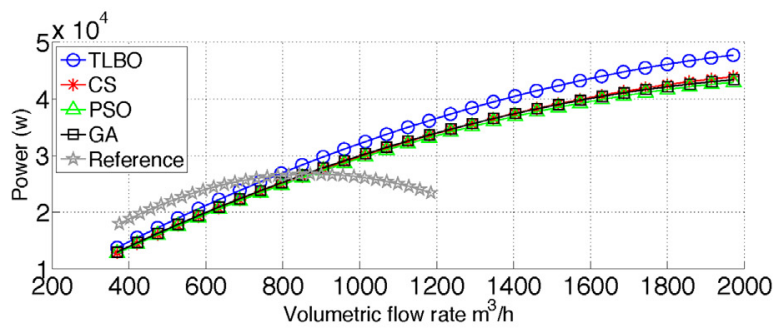

(e) Evolution of the power

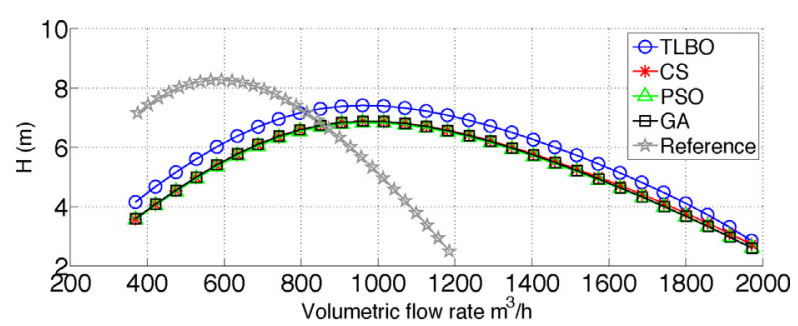

(b) Evolution of the head

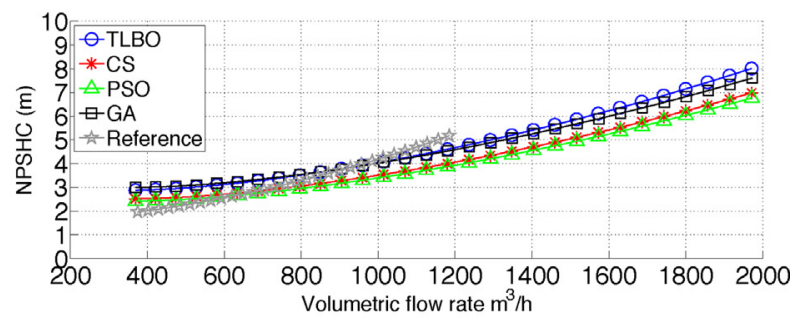

(d) Evolution of the NPSHC

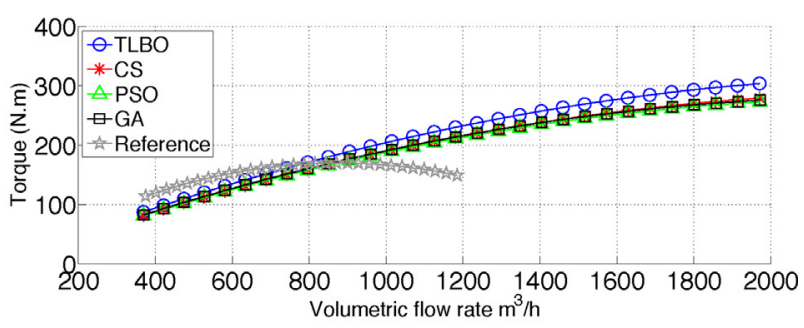

(f) Evolution of the torque

Fig. 24. Case 2-scenario 1: performances.

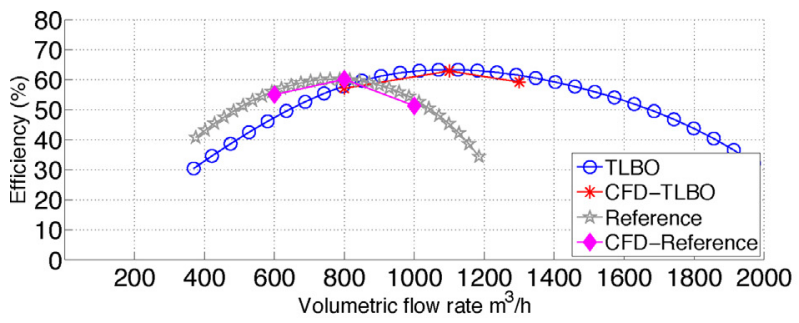

(a) Evolution of total efficiency

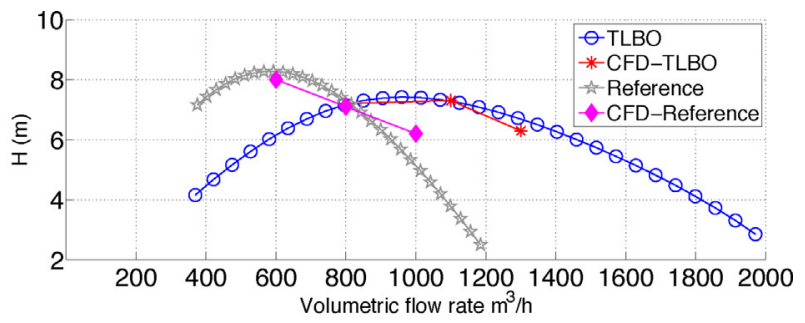

(b) Evolution of the head

Fig. 25. Comparison of the performance prediction of proposed approach with CFD: case 2-scenario 1.
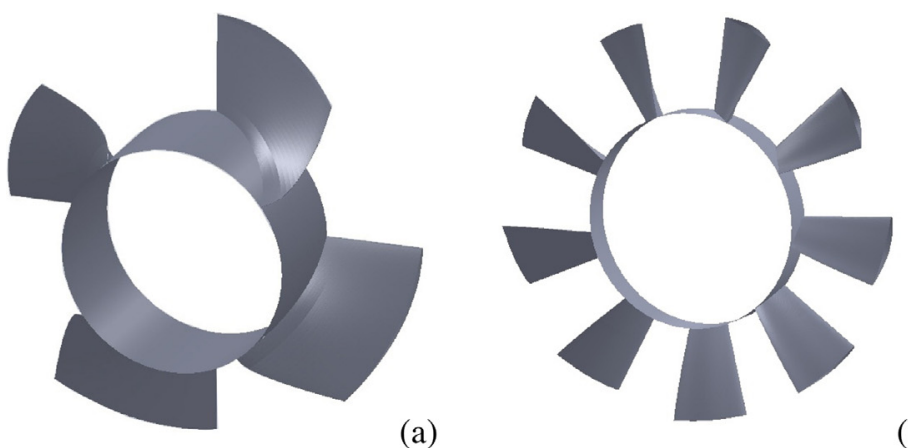

(b)

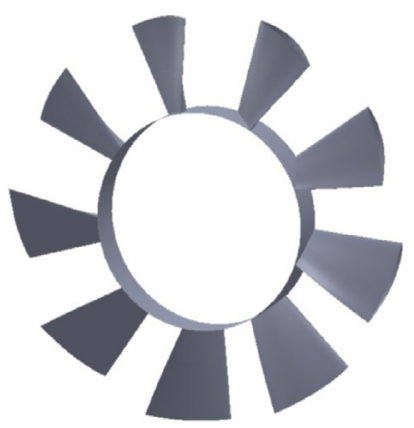

(c)

Fig. 26. Case 2-scenario 2: (a) reference rotor CAD, (b) PSO rotor CAD and (c) TLBO rotor CAD. 


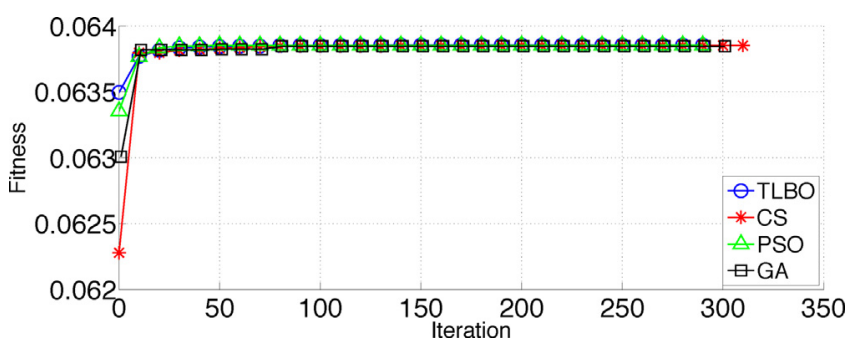

Fig. 27. Comparison of best fitness's variation with the iterations: case 2-scenario

scenario and about $27.6 \%$ for the second. In this comparison of the two objectives, results are very close with a little improvement on the left of design point for the second objective.

Another comparison with pump B of Ref. [25] is shown in Table 25. The hydraulic efficiency improvement is about $7 \%$ and $18 \%$ for the scenarios S1 and S2 respectively. Fig. 32 displays a good distribution of hydraulic efficiency with great precision in design point, (i.e. $0.6 \mathrm{~m}^{3} / \mathrm{s}$ ) and wide range.

Last comparison is carried out for pump $\mathrm{C}$ of latter reference. The pump is of the rotor-stator type and we focus only on rotor optimization, i.e. during the design optimization, the stator parameters design is kept constant with the same value of that of the reference pump. Despite that, the proposed approach recorded the improvement of the efficiency with about $6.74 \%$ and $8.33 \%$ for the scenarios S1 and S2 respectively, see Table 26 . The wide operating range is remarkable in Fig. 33. The design could be further improved inclunding the stator parameters in variables design.

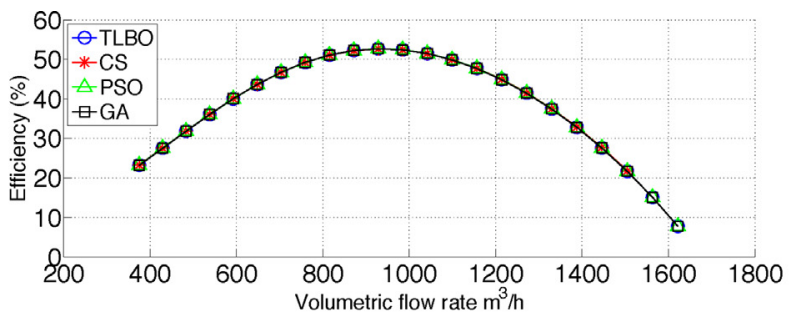

(a) Evolution of total efficiency

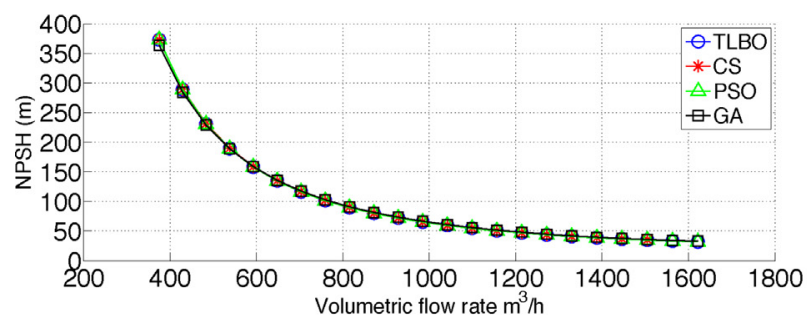

(c) Evolution of the NPSH

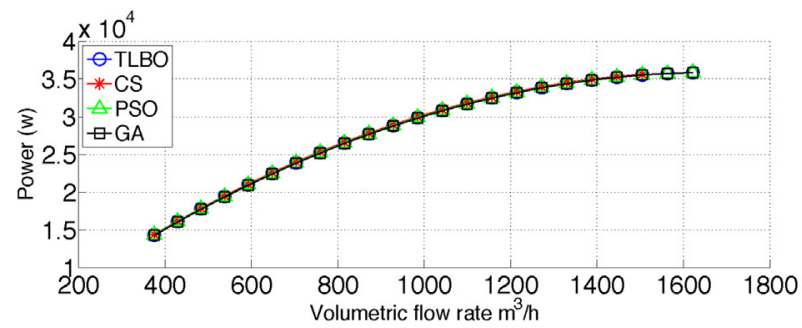

(e) Evolution of the power

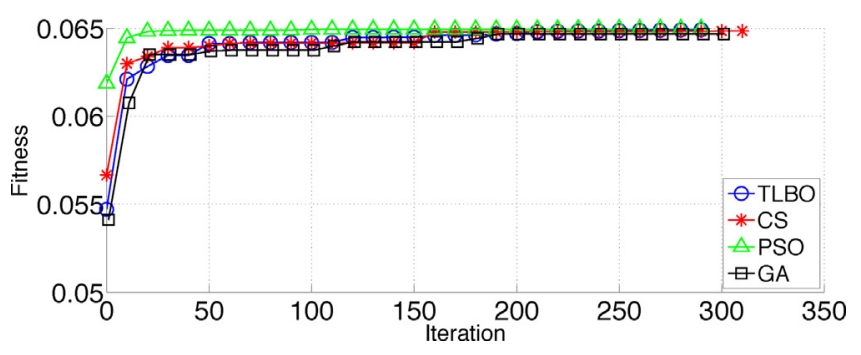

Fig. 29. Comparison of best fitness's variation with the iterations: case 2-scenario 3.

Numerically, these comparisons prove the effectiveness of the proposed approach in incompressible axial turbomachine with any operating condition (head, rotational speed, volume flow rate).

\section{Conclusion}

In this paper an assessment study of some meta-heuristics applied to the design optimization of incompressible axial turbomachines is performed. A design strategy based on the inverse and direct approaches was developed using bio and socio-inspired algorithms as well as a local search algorithm. The aim is to improve the volume flow rate efficiency while respecting a large number of constraints, it is improved about $10 \pm 0.288 \%$ in case 1 -scenario 1 and about $14 \%$ in case 1 -scenario 5 . Numerically, the results in the second formulation show that the range of efficiency/flow rate can be increased widely (about $60 \pm 0.669 \%$ in case 2 -scenario 1 ) with a little increase of nominal efficiency of reference machine. Besides,

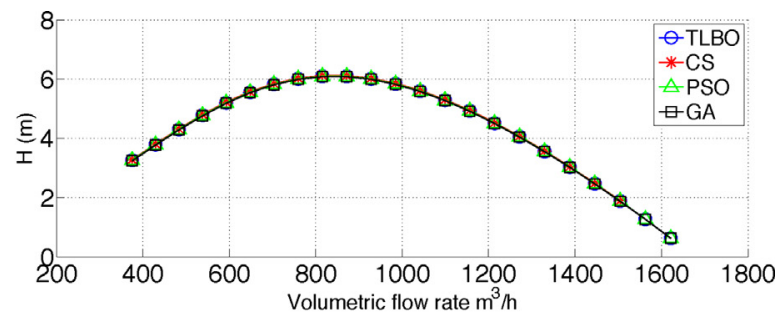

(b) Evolution of the head

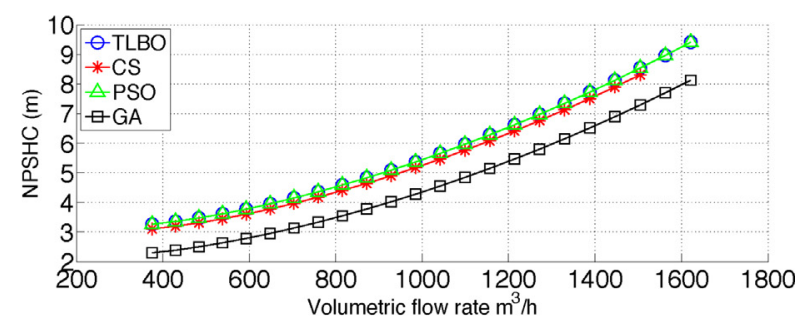

(d) Evolution of the NPSHC

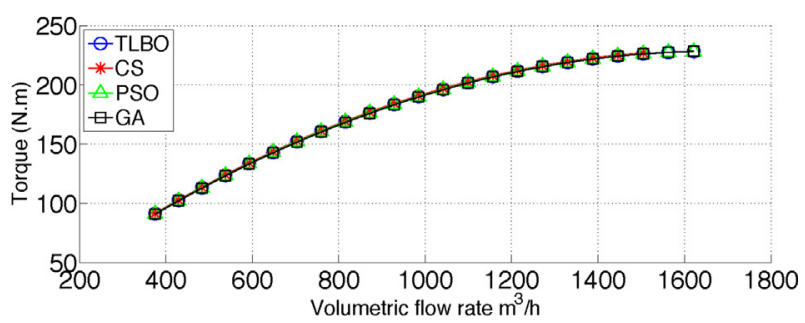

(f) Evolution of the torque

Fig. 28. Case 2-scenario 2: performances. 
Table 21

Results of different methods: case 2-scenario 3.

\begin{tabular}{|c|c|c|c|c|c|c|c|c|}
\hline \multirow[t]{2}{*}{$F$} & \multicolumn{4}{|l|}{ TLBO } & \multicolumn{4}{|l|}{$\mathrm{CS}$} \\
\hline & best & worst & ave & std & best & worst & ave & std \\
\hline Area & 0.0649398 & 0.0642566 & 0.0644795 & $2.8594 \mathrm{e}-04$ & 0.0648519 & 0.064356 & 0.06456078 & $1.4932 \mathrm{e}-04$ \\
\hline \multirow[t]{2}{*}{$F$} & \multicolumn{4}{|l|}{ PSO } & \multicolumn{4}{|l|}{ GA } \\
\hline & best & worst & ave & std & best & worst & ave & std \\
\hline Area & 0.0649804 & 0.0648374 & 0.06494251 & $4.2905 e-05$ & 0.0646773 & 0.0641161 & 0.06442932 & $1.9858 \mathrm{e}-04$ \\
\hline
\end{tabular}

Table 22

Comparison of the optimal solutions: case 2-scenario 3.

\begin{tabular}{|c|c|c|c|c|c|c|}
\hline Methods & $N(\mathrm{rpm})$ & $R_{e}^{1}(\mathrm{~m})$ & $R_{e}^{2}(\mathrm{~m})$ & $D_{i}$ & $D_{e}$ & $Z$ \\
\hline TLBO & 1849 & 0.1523672 & 0.153993 & 0.698896 & 0.501064 & 4 \\
\hline CS & 1856 & 0.152128 & 0.1538 & 0.694437 & 0.500728 & 4 \\
\hline PSO & 1899 & 0.152123 & 0.153882 & 0.699758 & 0.470388 & 3 \\
\hline GA & 1841 & 0.149659 & 0.151783 & 0.696984 & 0.495498 & 5 \\
\hline
\end{tabular}

Table 23

Performance and stability ranking of algorithms for all cases studied.

\begin{tabular}{|c|c|c|c|c|c|c|c|c|c|c|c|c|c|c|c|c|}
\hline \multirow[t]{3}{*}{ Methods } & \multirow[t]{3}{*}{ Fitness } & \multicolumn{8}{|c|}{ Case 1} & \multicolumn{2}{|c|}{ Case 2} & \multirow{3}{*}{$\begin{array}{l}\text { S3 } \\
\text { CV }\end{array}$} & \multirow[t]{3}{*}{ Sum } & \multirow[t]{3}{*}{ Rank } & \multirow[t]{3}{*}{ Average rank } & \multirow[t]{3}{*}{ Final rank } \\
\hline & & \multirow{2}{*}{$\begin{array}{l}\text { S1 } \\
\text { FV }\end{array}$} & \multirow{2}{*}{$\begin{array}{l}\text { S2 } \\
\text { FV }\end{array}$} & \multirow{2}{*}{$\begin{array}{l}\text { S3 } \\
\text { FV }\end{array}$} & \multicolumn{2}{|l|}{ S4 } & \multicolumn{3}{|l|}{ S5 } & \multirow{2}{*}{$\frac{\mathrm{S} 1}{\mathrm{CV}}$} & \multirow{2}{*}{$\begin{array}{l}\mathrm{S} 2 \\
\mathrm{CV}\end{array}$} & & & & & \\
\hline & & & & & $\mathrm{CV}$ & FCV & FV & $\mathrm{CV}$ & FCV & & & & & & & \\
\hline \multirow[t]{4}{*}{ TLBO } & best & 2 & 1 & 1 & 3 & 1 & 1 & 1 & 1 & 2 & 1 & 2 & 16 & 2 & \multirow[t]{4}{*}{1.25} & \multirow[t]{4}{*}{1} \\
\hline & worst & 1 & 3 & 2 & 1 & 1 & 1 & 1 & 1 & 1 & 1 & 3 & 16 & 1 & & \\
\hline & ave & 1 & 1 & 2 & 1 & 1 & 1 & 1 & 1 & 1 & 2 & 3 & 15 & 1 & & \\
\hline & std & 1 & 3 & 3 & 1 & 2 & 1 & 1 & 1 & 1 & 1 & 4 & 19 & 1 & & \\
\hline \multirow[t]{4}{*}{$\mathrm{CS}$} & best & 3 & 3 & 3 & 1 & 3 & 1 & 1 & 1 & 3 & 3 & 3 & 25 & 3 & \multirow[t]{4}{*}{2.25} & \multirow[t]{4}{*}{2} \\
\hline & worst & 3 & 1 & 3 & 3 & 1 & 1 & 1 & 1 & 3 & 3 & 2 & 22 & 2 & & \\
\hline & ave & 3 & 2 & 3 & 3 & 3 & 1 & 1 & 1 & 2 & 3 & 2 & 24 & 2 & & \\
\hline & std & 3 & 1 & 2 & 3 & 4 & 1 & 1 & 1 & 3 & 3 & 2 & 24 & 2 & & \\
\hline \multirow[t]{4}{*}{ PSO } & best & 1 & 1 & 1 & 3 & 1 & 1 & 1 & 1 & 1 & 1 & 1 & 13 & 1 & \multirow[t]{4}{*}{2.5} & \multirow[t]{4}{*}{3} \\
\hline & worst & 2 & 2 & 1 & 1 & 1 & 1 & 4 & 4 & 4 & 2 & 1 & 23 & 3 & & \\
\hline & ave & 2 & 3 & 1 & 2 & 2 & 1 & 4 & 4 & 4 & 1 & 1 & 25 & 3 & & \\
\hline & std & 2 & 2 & 1 & 2 & 3 & 1 & 4 & 4 & 4 & 2 & 1 & 26 & 3 & & \\
\hline \multirow[t]{4}{*}{ GA } & best & 4 & 4 & 4 & 1 & 4 & 4 & 4 & 4 & 4 & 4 & 4 & 41 & 4 & \multirow[t]{4}{*}{4} & \multirow[t]{4}{*}{4} \\
\hline & worst & 4 & 4 & 4 & 4 & 4 & 4 & 3 & 3 & 2 & 4 & 4 & 40 & 4 & & \\
\hline & ave & 4 & 4 & 4 & 4 & 4 & 4 & 3 & 3 & 3 & 4 & 4 & 41 & 4 & & \\
\hline & std & 4 & 4 & 4 & 4 & 1 & 4 & 3 & 3 & 2 & 4 & 3 & 36 & 4 & & \\
\hline
\end{tabular}

Table 24

Comparison of the optimal solution: pump A [50], $N=1400 \mathrm{rpm}, Q_{v}=1000 \mathrm{~m}^{3} / \mathrm{h}, H=8 \mathrm{~m}$.

\begin{tabular}{|c|c|c|c|c|c|c|c|c|c|c|}
\hline Methods & Efficiency (\%) & Area & $H(\mathrm{~m})$ & $R_{i}^{1}(\mathrm{~m})$ & $R_{e}^{1}(\mathrm{~m})$ & $R_{i}^{2}(\mathrm{~m})$ & $R_{e}^{2}(\mathrm{~m})$ & $D_{i}$ & $D_{e}$ & $Z$ \\
\hline Reference & 65 & 0.0695 & 7.812 & 0.0705 & 0.157 & 0.0705 & 0.157 & - & - & 6 \\
\hline TLBO C1 S1 & 65.78 & - & 8.00 & 0.0300249 & 0.149684 & 0.0613356 & 0.157 & 0.699 & 0.677 & 3 \\
\hline TLBO C2 S1 & - & 0.08867 & 8.00 & 0.0308582 & 0.149755 & 0.062324 & 0.157 & 0.699999 & 0.681804 & \\
\hline TLBO C1 S2 & 72.13 & - & 8.00 & 0.0344461 & 0.169401 & 0.0667512 & 0.172699 & 0.699 & 0.638 & 3 \\
\hline TLBO C2 S2 & - & 0.09637 & 8.00 & 0.0416876 & 0.168948 & 0.0721562 & 0.172755 & 0.699989 & 0.6204773 & 3 \\
\hline
\end{tabular}

Table 25

Comparison of the optimal solution: pump B [25], $N=1480 \mathrm{rpm}, Q_{v}=2160 \mathrm{~m}^{3} / \mathrm{h}, H=6 \mathrm{~m}$.

\begin{tabular}{|c|c|c|c|c|c|c|c|c|}
\hline Methods & Hydraulic efficiency (\%) & $R_{i}^{1}(\mathrm{~m})$ & $R_{e}^{1}(\mathrm{~m})$ & $R_{i}^{2}(\mathrm{~m})$ & $R_{e}^{2}(\mathrm{~m})$ & $D_{i}$ & $D_{e}$ & $Z$ \\
\hline Reference & 75 & 0.68 & 0.184 & 0.68 & 0.184 & 0.26 & 0.34 & 5 \\
\hline TLBO S1 & 82.90 & 0.03 & 0.182813 & 0.03 & 0.184 & 0.214246 & 0.699 & 3 \\
\hline TLBO S2 & 93.55 & 0.03 & 0.201887 & 0.03 & 0.2024 & 0.213066 & 0.699 & 3 \\
\hline
\end{tabular}

Table 26

Comparison of the optimal solution: pump C [25], $N=980 \mathrm{rpm}, Q_{v}=2160 \mathrm{~m}^{3} / \mathrm{h}, H=6 \mathrm{~m}$.

\begin{tabular}{|c|c|c|c|c|c|c|c|c|}
\hline Methods & Hydraulic efficiency (\%) & $R_{i}^{1}(\mathrm{~m})$ & $R_{e}^{1}(\mathrm{~m})$ & $R_{i}^{2}(\mathrm{~m})$ & $R_{e}^{2}(\mathrm{~m})$ & $D_{i}$ & $D_{e}$ & $Z$ \\
\hline Reference & 86 & 0.105 & 0.217 & 0.105 & 0.217 & 0.5 & 0.3 & 5 \\
\hline TLBO S1 & 92.74 & 0.0750967 & 0.217 & 0.0750967 & 0.217 & 0.406172 & 0.7 & 8 \\
\hline TLBO S2 & 94.33 & 0.0336081 & 0.238697 & 0.0415088 & 0.238581 & 0.513954 & 0.580032 & 5 \\
\hline
\end{tabular}




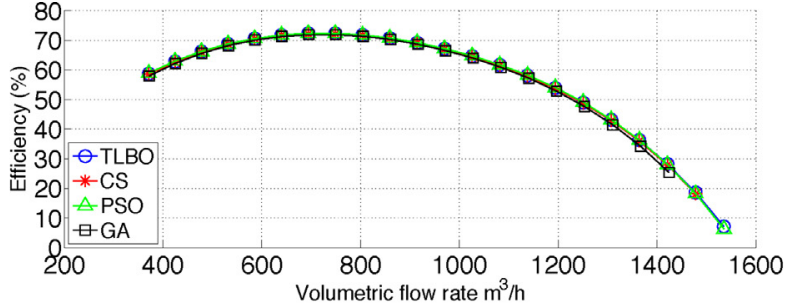

(a) Evolution of total efficiency

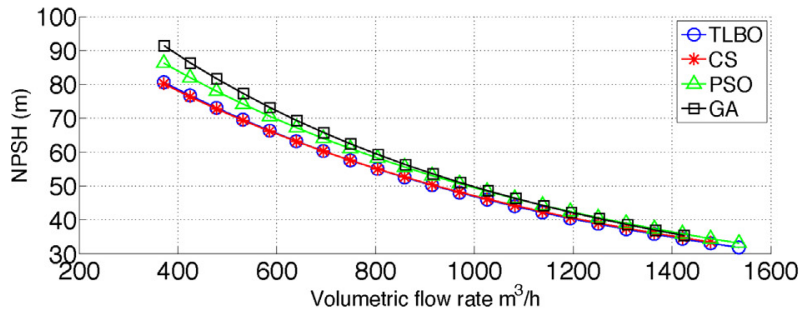

(c) Evolution of the NPSH

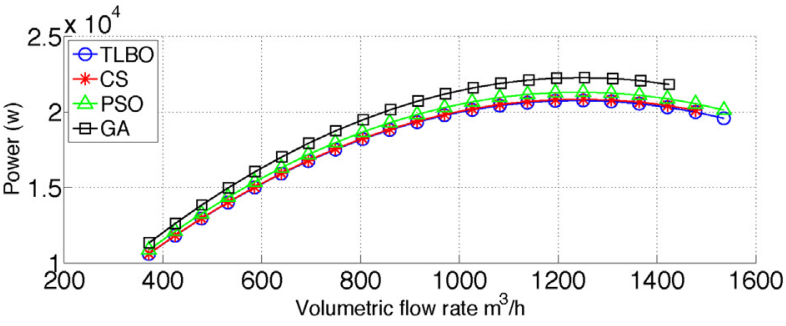

(e) Evolution of the power

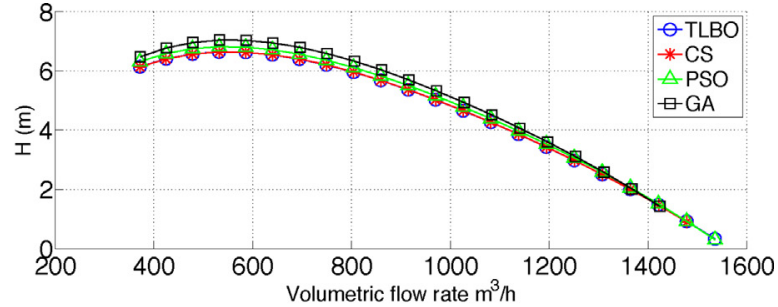

(b) Evolution of the head

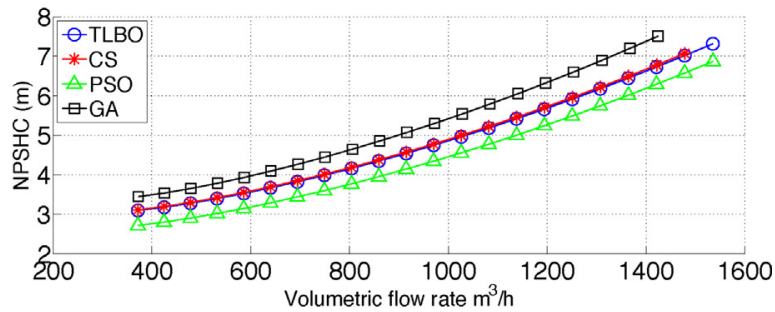

(d) Evolution of the NPSHC

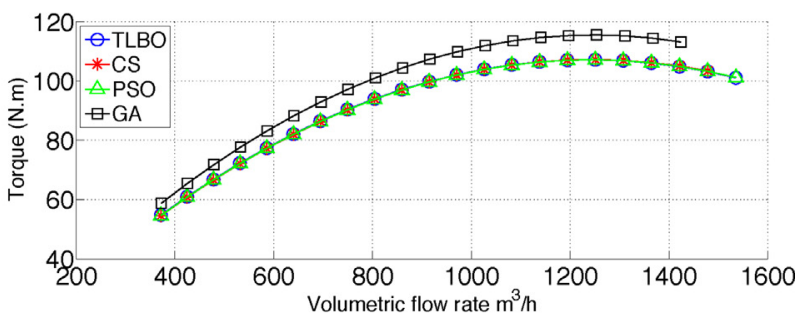

(f) Evolution of the torque

Fig. 30. Case 2-scenario 3: performances.

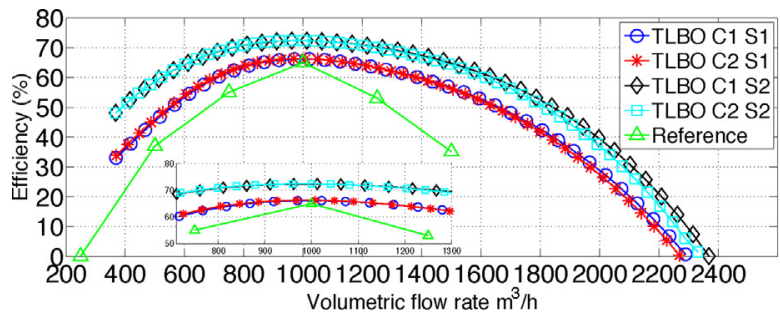

(a) Evolution of total efficiency

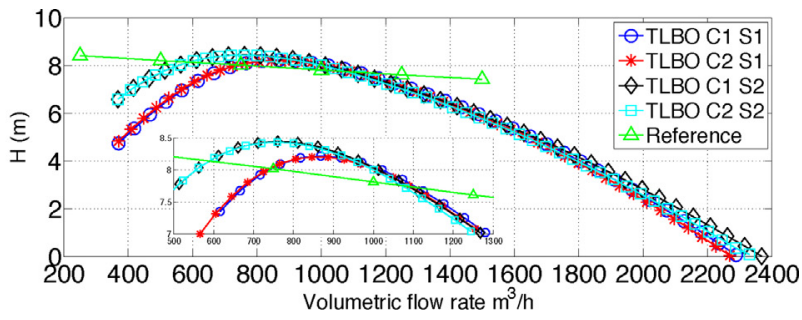

(b) Evolution of the head

Fig. 31. Pump A: performances.

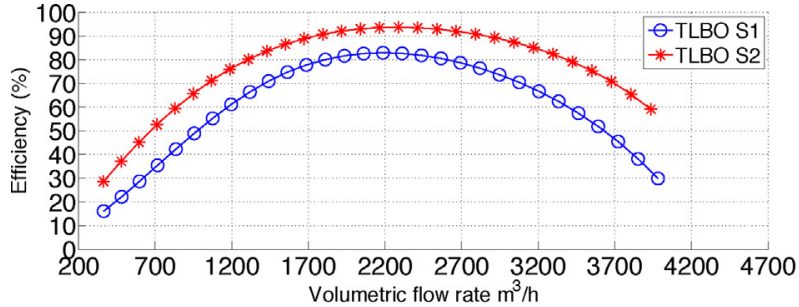

(a) Evolution of total efficiency

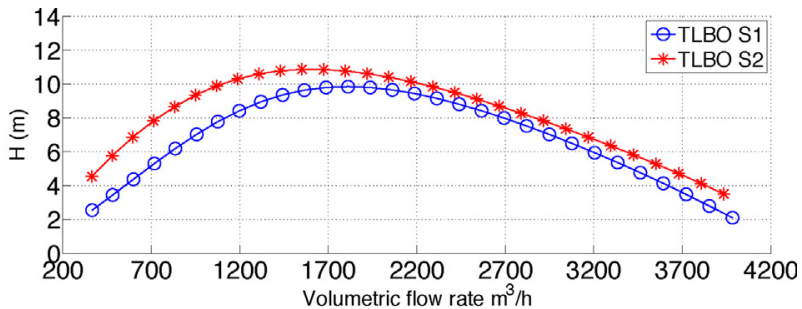

(b) Evolution of the head

Fig. 32. Pump B: performances.

an improvement of the efficiency was highlighted with regard to the radius at the rotor inlet and outlet, also expressed when the exterior radius is high and the interior radius is low. The best con- figuration is when all radius are considered variables, this is due to the extension of side constraints.

A comparative study allowed us to highlight the robustness, the flexibility and the implementation ease of the meta-heuristics 


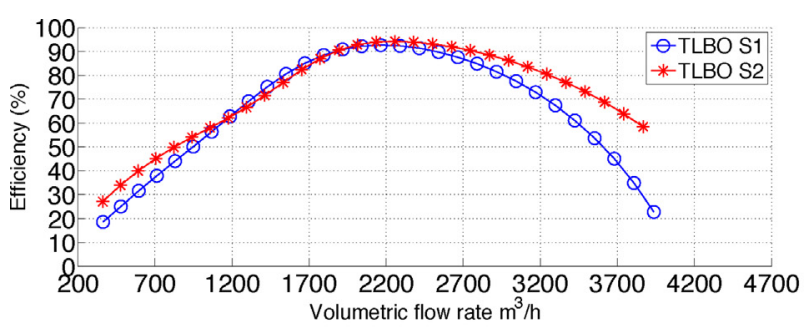

(a) Evolution of total efficiency

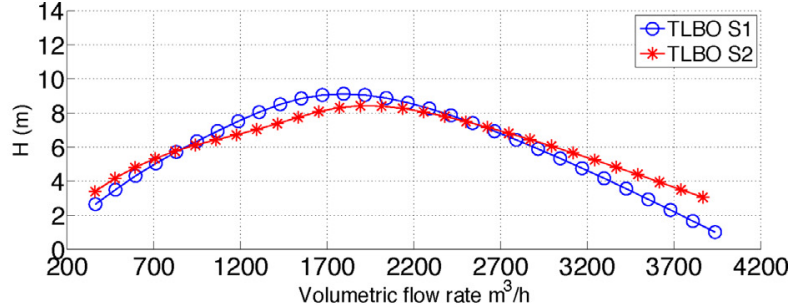

(b) Evolution of the head

Fig. 33. Pump C: performances.

in terms of efficiency improvement of turbomachines. The TLBO algorithm can be used efficiently for turbomachinery design that includes a high number of several constraint types. For constraint handling, the modified penalty technique can dramatically decrease the computational time. With a large number of constraints, the optimization algorithms behave differently. The algorithm without the controlling parameters is the most profitable in this kind of optimization problem. It is proved also that the TLBO, CS and PSO are more suitable than GA and SLP for the problem treated in this work.

As an outlook, the design can be more improved with the consideration of other criteria in the context of a multi-objective optimization approach by the implementation of the same metaheuristics. The computational time may be reduced substantially by the integration of substituted models, where the variables will be directly connected to the objective function and to the considered constraints.

\section{References}

[1] A.J. Stepanoff, Centrifugal and Axial Flow Pumps: Theory, Design, and Application, Krieger Publishing Company, 1957.

[2] J.P. Veres, Centrifugal and Axial Pump Design and Off-Design Performance Prediction, National Aeronautics and Space Administration, 1994.

[3] Z. Dugao, Z. Jiang, Optimization design of an axial-flow fan used for mining local-ventilation, Comput. Ind. Eng. 31 (3) (1996) 691-696.

[4] D. Desai, J. Zhou, Optimization design of an axial-flow fan used in local ventilation in mining industry, in: ASME 2011 International Mechanical Engineering Congress and Exposition, American Society of Mechanical Engineers, 2011, pp. 879-883.

[5] K. OH, HW, K. KIM, Conceptual design optimization of mixed-flow pump impellers using mean streamline analysis, Proc. Inst. Mech. Eng. Part A: J. Power Energy 215 (A1) (2001) 133-138.

[6] D. Sorensen, J. Sorensen, Toward improved rotor-only axial fans - Part I: A numerically efficient aerodynamic model for arbitrary vortex flow, J. Fluids Eng. 122 (2) (2000) 318-323.

[7] D. Sorensen, M. Thompson, J. Sorensen, Toward improved rotor-only axial fans - Part II: Design optimization for maximum efficiency, J. Fluids Eng. 122 (2) (2000) 324-329.

[8] E. Benini, A. Toffolo, A parametric method for optimal design of two-dimensional cascades, Proc. Inst. Mech. Eng. Part A: J. Power Energy 215 (4) (2001) 465-473.

[9] A. Oyama, M.-S. Liou, S. Obayashi, Transonic axial-flow blade optimization: evolutionary algorithms/three-dimensional Navier-Stokes solver, J. Propuls. Power 20 (4) (2004) 612-619.

[10] C.-h. Cho, S.-y. Cho, C. Kim, Development of an axial-type fan with an optimization method, Front. Energy Power Eng. China 3 (4) (2009) 414-422.

[11] W. Tiow, K.F.C. Yiu, M. Zangeneh, Application of simulated annealing to inverse design of transonic turbomachinery cascades, Proc. Inst. Mech. Eng. Part A: J. Power Energy 216 (1) (2002) 59-73.

[12] T.J. Carrigan, B.H. Dennis, Z.X. Han, B.P. Wang, Aerodynamic shape optimization of a vertical-axis wind turbine using differential evolution, ISRN Renew. Energy 2012 (2012) 1-16.

[13] B.H. Dennis, G.S. Dulikravich, Z.-X. Han, Optimization of turbomachinery airfoils with a genetic/sequential-quadratic-programming algorithm, J. Propuls. Power 17 (5) (2001) 1123-1128.

[14] S. Shahpar, A comparative study of optimisation methods for aerodynamic design of turbomachinery blades, ASME Paper (2000-GT) (2000) 523

[15] A. Safari, K. Hajikolaei, H. Lemu, G. Wang, A high-dimensional model representation guided PSO methodology with application on compressor airfoil shape optimization, in: ASME Turbo Expo 2016: Turbomachinery Technical Conference and Exposition, American Society of Mechanical Engineers, 2016, p. V02CT45A013.
[16] D. Yanhui, W. Wenhua, F. Zhaolin, C. Ti, An introduction of aerodynamic shape optimization platform for compressor blade, in: ASME Turbo Expo 2016: Turbomachinery Technical Conference and Exposition, American Society of Mechanical Engineers, 2016, p. V02CT39A031.

[17] Z. Li, X. Zheng, Review of design optimization methods for turbomachinery aerodynamics, Prog. Aerosp. Sci. 93 (2017) 1-23.

[18] W.-G. Li, NPSHr optimization of axial-flow pumps, J. Fluids Eng. 130 (7) (2008) 074504.

[19] B.-J. Lin, C.-I. Hung, E. Tang, An optimal design of axial-flow fan blades by the machining method and an artificial neural network, Proc. Inst. Mech. Eng. Part C: J. Mech. Eng. Sci. 216 (3) (2002) 367-376.

[20] C.-H. Huang, C.-W. Gau, An optimal design for axial-flow fan blade: theoretical and experimental studies, J. Mech. Sci. Technol. 26 (2) (2012) 427-436.

[21] K.-Y. Lee, Y.-S. Choi, Y.-L. Kim, J.-H. Yun, Design of axial fan using inverse design method, J. Mech. Sci. Technol. 22 (10) (2008) 1883-1888.

[22] K.-S. Lee, K.-Y. Kim, A. Samad, Design optimization of low-speed axial flow fan blade with three-dimensional RANS analysis, J. Mech. Sci. Technol. 22 (10) (2008) 1864-1869.

[23] P. Song, J. Sun, Blade shape optimization for transonic axial flow fan, J. Mech. Sci. Technol. 29 (3) (2015) 931-938.

[24] R. Robert, N. Ricardo, B. Farid, Pompes rotodynamiques aérohydrodynamique des profils et aubages de pompes hélices, Techniques de l'Ingénieur, Réf. BM4304 V1.

[25] R. Robert, N. Ricardo, B. Farid, Pompes rotodynamiques - dimensionnement et analyse des performances des pompes hélices, Techniques de l'Ingénieur, Réf. BM4305.

[26] K. Deb, Multi-objective Optimization Using Evolutionary Algorithms, vol. 16, John Wiley \& Sons, 2001.

[27] A. Ponsich, C. Azzaro-Pantel, S. Domenech, L. Pibouleau, Constraint handling strategies in genetic algorithms application to optimal batch plant design, Chem. Eng. Process.: Process Intensif. 47 (3) (2008) 420-434.

[28] K. Deb, An efficient constraint handling method for genetic algorithms, Comput. Methods Appl. Mech. Eng. 186 (2) (2000) 311-338.

[29] A.E. Smith, D.W. Coit, T. Baeck, D. Fogel, Z. Michalewicz, Penalty functions Handbook of Evolutionary Computation, vol. 97 (1, 1995, pp. C5.

[30] J.H. Holland, Adaptation in Natural and Artificial Systems: An Introductory Analysis with Applications to Biology, Control, and Artificial Intelligence, vol. viii, Univ. Michigan Press, Oxford, England, 1975.

[31] D. Goldberg, in: N.N. Schraudolph (Ed.), Genetic Algorithms in Search, Optimization, and Machine Learning, vol. 3, Addison-Wesley, Reading, MA, 1989, p. 1.

[32] Z. Michalewicz, Genetic Algorithms + Data Structures = Evolution Programs, Springer Science \& Business Media, 1996.

[33] Y. Kaya, M. Uyar, et al., A Novel Crossover Operator for Genetic Algorithms: Ring Crossover, 2011 arXiv:1105.0355.

[34] L. Song, NGPM - A NSGA-II Program in Matlab, College of Astronautics, North Western Polytechnical University, China, 2011.

[35] J. Kenndy, R. Eberhart, Particle swarm optimization, Proceedings of IEEE International Conference on Neural Networks, vol. 4 (1995) 1942-1948.

[36] Y. Shi, R. Eberhart, A modified particle swarm optimizer, Evolutionary Computation Proceedings, 1998. IEEE World Congress on Computationa Intelligence. The 1998 IEEE International Conference on IEEE (1998) 69-73.

[37] F. Van Den Bergh, An Analysis of Particle Swarm Optimizers, University of Pretoria, 2006 (Ph.D. thesis).

[38] J. Wei, L. Guangbin, L. Dong, Elite particle swarm optimization with mutation, System Simulation and Scientific Computing, 2008. ICSC 2008. Asia Simulation Conference - 7th International Conference on IEEE (2008) 800-803.

[39] X.-S. Yang, S. Deb, Cuckoo search via lévy flights, Nature \& Biologically Inspired Computing, 2009. NaBIC 2009. World Congress on IEEE (2009) 210-214.

[40] X.-S. Yang, Cuckoo search and firefly algorithm: overview and analysis, in: Cuckoo Search and Firefly Algorithm, Springer, 2014, pp. 1-26.

[41] X.-S. Yang, S. Deb, Engineering optimisation by cuckoo search, Int. J. Math. Model. Numer. Optim. 1 (4) (2010) 330-343.

[42] R.V. Rao, V.J. Savsani, D. Vakharia, Teaching-learning-based optimization: a novel method for constrained mechanical design optimization problems, Comput. Aided Des. 43 (3) (2011) 303-315. 
[43] R. Rao, V. Savsani, J. Balic, Teaching-learning-based optimization algorithm for unconstrained and constrained real-parameter optimization problems, Eng. Optim. 44 (12) (2012) 1447-1462.

[44] S.C. Satapathy, A. Naik, K. Parvathi, A teaching learning based optimization based on orthogonal design for solving global optimization problems, SpringerPlus 2 (1) (2013) 130.

[45] R. Fletcher, Quadratic Programming, Practical Methods of Optimization, second edition, 2000, pp. 229-258.

[46] M.S. Bazaraa, H. Sherali, C. Shetty, Nonlinear Programming: Theory and Algorithms, John Wiley \& Sons, New York, 1993.
[47] T.-Y. Chen, Calculation of the move limits for the sequential linear programming method, Int. J. Numer. Methods Eng. 36 (15) (1993) 2661-2679

[48] L. Lamberti, C. Pappalettere, Move limits definition in structural optimization with sequential linear programming. Part I: Optimization algorithm, Comput. Struct. 81 (4) (2003) 197-213.

[49] G. Dantzig, M. Thapa, Linear Programming 1: Introduction (Springer Series in Operations Research and Financial Engineering), 1997.

[50] Manjunatha, J.R. Nataraj, Design and analysis of impeller blade for axial flow pumps, Int. J. Eng. Res. Manag. Stud. (2015) 1-31. 